\title{
Obstructions for gluing biset functors
}

\author{
Olcay Coşkun ${ }^{a}$, Ergün Yalçın ${ }^{\text {b,* }}$ \\ a Department of Mathematics, Boğaziçi University, 34342 Bebek, Istanbul, Turkey \\ b Department of Mathematics, Bilkent University, 06800 Bilkent, Ankara, Turkey
}

\section{A R T I C L E I N F O}

Article history:

Received 26 November 2018

Available online 3 June 2019

Communicated by Markus

Linckelmann

\section{Keywords:}

Biset functors

Dade group

Higher limits

Quillen category

\section{A B S T R A C T}

We develop an obstruction theory for the existence and uniqueness of a solution to the gluing problem for a biset functor defined on the subquotients of a finite group $G$. The obstruction groups for this theory are the reduced cohomology groups of a category $\mathcal{D}_{G}^{*}$ whose objects are the sections $(U, V)$ of $G$, where $1 \neq V \unlhd U \leq G$, and whose morphisms are defined as a generalization of morphisms in the orbit category. Using this obstruction theory, we calculate the obstruction group for some well-known $p$-biset functors, such as the Dade group functor defined on $p$-groups with $p$ odd.

(c) 2019 Elsevier Inc. All rights reserved.

\section{Introduction and definitions}

Given a commutative ring $R$ with unity, the $R$-biset category $R \mathcal{B}$ is a category whose objects are all finite groups, and in which the morphisms from the finite group $H$ to the finite group $K$ are given by the Burnside module $R \otimes_{\mathbb{Z}} B(K, H)$ of $(K, H)$-bisets, or equivalently of left $K \times H$-sets. The composition in the biset category is given by the composition product of bisets (see Section 2 for details). An $R$-linear functor

\footnotetext{
* Corresponding author.

E-mail addresses: olcay.coskun@boun.edu.tr (O. Coşkun), yalcine@fen.bilkent.edu.tr (E. Yalçın).
} 


$$
F: R \mathcal{B} \rightarrow R \text {-mod }
$$

is called a biset functor over $R$. The Burnside ring functor $B$ and the representation ring functor $R_{K}$ defined on finite groups, and the Dade group $D$ defined on $p$-groups with $p$ odd, are important examples of biset functors.

Let $G$ be a finite group. A pair $(U, V)$ of subgroups of $G$ is called a section of $G$ if $V \unlhd U \leq G$. The quotient group $U / V$ is called a subquotient of $G$. In the literature sometimes these two terms are used in an equivalent way. Our convention will be as follows: Let $\mathcal{X}^{s}$ denote the set of all quotient groups $U / V$ indexed by the set of sections $(U, V)$ of $G$. We denote the $R$-biset category over the collection $\mathcal{X}^{s}$ by $R \mathcal{B}_{G}$. An $R$-linear functor $F: R \mathcal{B}_{G} \rightarrow R$-mod is called a biset functor for $G$ over $R$.

Every $(K, H)$-biset can be expressed as a composition of five types of bisets, called induction, inflation, isogation, deflation, and restriction bisets. Descriptions of these bisets can be found in [3, 2.3.9] (isogation is a biset associated to an isomorphism, see [1, pg. 3811]). For $V \unlhd U \leq G$, the composition of deflation and restriction maps $\operatorname{Res}_{U}^{G}$ and $\operatorname{Def}_{U / V}^{U}$ is often denoted by $\operatorname{Defres}_{U / V}^{G}$ or $\operatorname{Des}_{U / V}^{G}$, and is called the destriction biset. Similar to biset functors, one can also define a notion of destriction functor, which uses only the destriction maps and isogations induced by conjugations. Every biset functor can be considered as a destriction functor via the algebra map from the destriction algebra to the alchemic algebra over the collection $\mathcal{X}^{s}$ (see Section 2.2 for details).

For every $K \unlhd H \leq G$, the normalizer of the section $(H, K)$ is defined as the subgroup $N_{G}(H, K)=N_{G}(H) \cap N_{G}(K)$.

Definition 1.1. Let $F$ be a biset functor or a destriction functor for $G$ over $R$. A gluing data for $F(G)$ is a sequence $\left(f_{H}\right)_{1<H \leq G}$ of elements $f_{H} \in F\left(N_{G}(H) / H\right)$ satisfying the following compatibility conditions:

(i) (Conjugation invariance) For any $g \in G$ and $H \leq G$, we have

$$
{ }^{g} f_{H}=f_{g_{H}} .
$$

(ii) (Destriction invariance) For any $K \unlhd H \leq G$, we have

$$
\operatorname{Defres}_{N_{G}(H, K) / H}^{N_{G}(K) / K} f_{K}=\operatorname{Res}_{N_{G}(H, K) / H}^{N_{G}(H) / H} f_{H} .
$$

The gluing problem for the biset functor $F$ at $G$ is the problem of finding an element $f \in F(G)$ such that for any non-trivial subgroup $H$ of $G$, we have $\operatorname{Defres}_{N_{G}(H) / H}^{G} f=f_{H}$. If such an element exists, we call it a solution to the given gluing data.

The gluing problem was first introduced for endo-permutation modules by Bouc and Thévenaz [6]. When a solution to a gluing problem exists we would like to also know if it is unique. Following Bouc and Thévenaz [6], we denote the set of all gluing data for $F(G)$ as the inverse limit 


$$
\lim _{1<\overleftarrow{H \leq G}} F\left(N_{G}(H) / H\right)
$$

Later we show that the gluing data is indeed an inverse limit over a category. The complete solution to the gluing problem is an exact sequence

$$
0 \longrightarrow \operatorname{Ker}\left(r_{G}^{F}\right) \longrightarrow F(G) \stackrel{r_{G}^{F}}{\longrightarrow} \lim _{1<H \leq G} F\left(N_{G}(H) / H\right) \longrightarrow \operatorname{Obs}(F(G)) \longrightarrow 0
$$

where $r_{G}^{F}$ denotes the map which takes $f \in F(G)$ to the tuple ( $\left.\operatorname{Defres}_{N_{G}(H) / H}^{G} f\right)_{1<H \leq G}$ and $\operatorname{Obs}(F(G))$ denotes the group of obstructions for a gluing data to have a solution.

Bouc and Thévenaz [6, Theorem 1.1] calculated the obstruction group for the torsion part of the Dade group $D_{t}(G)$ when $G$ is a noncyclic $p$-group with $p$ odd. They found that

$$
\operatorname{Obs}\left(D_{t}(G)\right) \cong H^{0}\left(\mathcal{A}_{\geq 2}(G) ; \mathbb{F}_{2}\right)^{G}
$$

where $\mathcal{A}_{\geq 2}(G)$ is the poset of elementary abelian subgroups of $G$ with rank $\geq 2$. Later Bouc [2, Theorem 2.15] showed that the obstruction group $\operatorname{Obs}(D(G))$ for the Dade group $D(G)$ of a $p$-group $G$, with $p$ odd, embeds into the group $H^{1}\left(\mathcal{A}_{\geq 2}(G) ; \mathbb{Z}\right)^{(G)}$ defined in [2, Notation 2.9] using $G$-invariant cocycles. We show in Lemma 7.7 that this group is isomorphic to the first cohomology group $H^{1}\left(\mathcal{A}_{\geq 2}(G) / G ; \mathbb{Z}\right)$ of the orbit space $\mathcal{A}_{\geq 2}(G) / G$.

Coşkun [8] extended these calculations to the biset functor for the dual of the rational representation ring $R_{\mathbb{Q}}^{*}$ and to the biset functor of Borel-Smith functions $C_{b}$. In all these calculations the results are expressed as modified versions of cohomology groups for some topological space. Our aim in this paper is to give a common framework for all these obstruction group calculations by expressing the obstruction group for a biset functor $F$ as the 0 -th reduced cohomology group of a category $\mathcal{D}_{G}$, which we introduce below. Using this theory we also obtain new results for the obstruction groups of certain $p$-biset functors such as the Dade group functor.

The set of sections of $G$ is a $G$-poset under the conjugation action $(U, V) \rightarrow\left({ }^{g} U,{ }^{g} V\right)$ of $G$, where ${ }^{g} U$ denotes the subgroup $g U g^{-1}$. The order relation is given by the inclusion of sections, that is, $(U, V) \preceq(M, L)$ if and only if $L \leq V \leq U \leq M$. We call a set of sections of $G$ a collection if it is closed under conjugation.

Definition 1.2. Let $\mathcal{C}$ be a collection of sections of a finite group $G$. The orbit category of sections $\mathcal{D}_{G}^{\mathcal{C}}$ is the category whose objects are sections $(U, V)$ in $\mathcal{C}$, and whose morphisms are given by

$$
\operatorname{Hom}_{\mathcal{D}_{G}^{c}}((U, V),(M, L))=\left\{M g \mid g \in G,{ }^{g}(U, V) \preceq(M, L)\right\} .
$$


If $N h:(M, L) \rightarrow(N, R)$ is a morphism in $\mathcal{D}_{G}^{\mathcal{C}}$, then the composition is given by $N h \circ$ $M g=N h g$. We denote the orbit category of sections over all sections of $G$ simply by $\mathcal{D}_{G}$.

Note that the composition defined above is well-defined: If $g^{\prime}=x g$ and $h^{\prime}=y h$ for some $x \in M$ and $y \in N$, then $N h^{\prime} g^{\prime}=N h x g=N h g$ because $h x h^{-1} \in{ }^{h} M \leq N$. We show that the category algebra for the opposite category of category $\mathcal{D}_{G}^{\mathcal{C}}$ is isomorphic to destriction algebra (see Definition 2.4 and Proposition 2.6). Using the algebra map from the destriction algebra to the alchemic algebra, we can consider a biset functor $F$ as an $R \mathcal{D}_{G}^{\mathcal{C}}$-module and study the gluing problem for $F$ using homological algebra over $R \mathcal{D}_{G}^{\mathcal{C}}$-modules. To simplify the notation we write $F(U / V)$ for $F((U, V))$ when $F$ is an $R \mathcal{D}_{G}$-module. We also write $F(G)$ for $F(G / 1)$ to simplify the notation further.

Given a collection of sections $\mathcal{C}$ of $G$, closed under taking subsections, let $T^{\mathcal{C}}$ denote the $R \mathcal{D}_{G}$-module whose values on those sections $(U, V) \notin \mathcal{C}$ are equal to $R$, and equal to zero on the sections in $\mathcal{C}$. Let $\underline{R}$ denote the constant functor of $\mathcal{D}_{G}$ and $J^{\mathcal{C}}$ denote the $R \mathcal{D}_{G}$-module such that $0 \rightarrow J^{\mathcal{C}} \rightarrow \underline{R} \rightarrow T^{\mathcal{C}} \rightarrow 0$ is an exact sequence. For a destriction functor or a biset functor $F$, this gives a long exact sequence

$$
0 \longrightarrow \operatorname{Hom}_{R \mathcal{D}_{G}}\left(T^{\mathcal{C}}, F\right) \longrightarrow F(G) \stackrel{r_{G}^{F}}{\longrightarrow} \lim _{(U, \overleftarrow{V}) \in \mathcal{D}_{G}^{\mathcal{C}}} F(U / V) \longrightarrow \operatorname{Ext}_{R \mathcal{D}_{G}}^{1}\left(T^{\mathcal{C}}, F\right) \longrightarrow 0
$$

which can be regarded as a general version of a gluing problem for a collection $\mathcal{C}$ that is closed under taking subsections. The obstruction group for this gluing problem over $\mathcal{C}$ is given by $\operatorname{Obs}^{\mathcal{C}}(F) \cong \operatorname{Ext}_{R \mathcal{D}_{G}}^{1}\left(T^{\mathcal{C}}, F\right)$.

We show that the gluing problem for $F$ stated in Definition 1.1 is a special case of this more general gluing problem over the category $\mathcal{D}_{G}^{\mathcal{C}}$.

Theorem 1.3. Let $F$ be a destriction functor or a biset functor for a finite group $G$ defined over R. Let $\mathcal{D}_{G}^{*}$ denote the orbit category of sections over the collection of all sections $(U, V)$ of $G$ with $V \neq 1$, and $T$ the constant functor on the set of all sections $(U, V)$ with $V=1$. Then there is an isomorphism

$$
\lim _{1<H \leq G} F\left(N_{G}(H) / H\right) \cong \lim _{(U, \overleftarrow{V}) \in \mathcal{D}_{G}^{*}} F(U / V)
$$

which gives isomorphisms

$$
\operatorname{Obs}(F(G)) \cong \operatorname{Ext}_{R \mathcal{D}_{G}}^{1}(T, F) \text { and } \operatorname{Ker}\left(r_{G}^{F}\right) \cong \operatorname{Hom}_{R \mathcal{D}_{G}}(T, F)
$$

This theorem is proved in Section 3 as Propositions 3.3 and 3.4. In Section 4, we discuss the gluing problem for an arbitrary collection $\mathcal{C}$ (not necessarily closed under taking subsections). We define the reduced cohomology $\widetilde{H}^{*}\left(\mathcal{D}_{G}^{\mathcal{C}} ; F\right)$ of the category $\mathcal{D}_{G}^{\mathcal{C}}$ and show that if the collection $\mathcal{C}$ is closed under taking subsections, then 


$$
\operatorname{Obs}^{\mathcal{C}}(F(G)) \cong \operatorname{Ext}_{\mathcal{D}_{G}}^{1}\left(T^{\mathcal{C}}, F\right) \cong \widetilde{H}^{0}\left(\mathcal{D}_{G}^{\mathcal{C}} ; F\right)
$$

To calculate these reduced cohomology groups, we apply a theorem of Jackowski and Slominska [13] on isotropy presheaves to the category $\mathcal{D}_{G}^{\mathcal{C}}$ and prove that under certain conditions the collection $\mathcal{C}$ can be replaced by a smaller collection $\mathcal{C}^{\prime}$ with isomorphic reduced cohomology group. When $G$ is a $p$-group, we show that the collection of all sections $(U, V)$ of $G$ with $V \neq 1$ can be reduced to the collections of all sections of the form $\left(C_{G}(E), E\right)$ where $E$ is a nontrivial elementary abelian subgroup of $G$.

This reduction allows us to relate the obstruction groups $\operatorname{Obs}(F(G))$ to the cohomology groups of the Quillen category $\mathcal{A}_{p}(G)$, the category whose objects are the nontrivial elementary abelian $p$-subgroups of $G$ and morphisms $E_{1} \rightarrow E_{2}$ are given by compositions of conjugation and inclusion maps. Given a destriction or biset functor $F$, there is an associated $R \mathcal{A}_{p}(G)$-module $\bar{F}$ defined by $\bar{F}(E)=F\left(C_{G}(E) / E\right)$ for every $E \in \mathcal{A}_{p}(G)$. We prove the following theorem.

Theorem 1.4. Let $G$ be a p-group and $\mathcal{A}_{p}(G)$ denote the Quillen category of $G$ over all nontrivial elementary abelian p-subgroups of $G$. Then, for a destriction functor or a biset functor $F$, there is an isomorphism

$$
\widetilde{H}^{*}\left(\mathcal{D}_{G}^{*} ; F\right) \cong \widetilde{H}^{*}\left(\mathcal{A}_{p}(G) ; \bar{F}\right) .
$$

In particular, $\operatorname{Obs}(F(G)) \cong \widetilde{H}^{0}\left(\mathcal{A}_{p}(G) ; \bar{F}\right)$.

As a consequence of Theorem 1.4 and a theorem of Oliver [15, Theorem 1], we also obtain that if $G$ is a $p$-group then

$$
\operatorname{Ext}_{R \mathcal{D}_{G}}^{n}(T, F) \cong \widetilde{H}^{n-1}\left(\mathcal{D}_{G}^{*} ; F\right)=0
$$

for $n \geq \operatorname{rk}(G)+1$, where $\operatorname{rk}(G)$ denotes the maximal rank of elementary abelian subgroups in $G$ (see Proposition 5.4).

In Section 6 we consider rhetorical $p$-biset functors and give an explicit formula for the obstruction groups $\operatorname{Obs}(F(G))$ for these functors (see Theorem 6.8). In Section 7, we apply this formula to gluing problems for various rhetorical $p$-biset functors. For the torsion part of the Dade group $D_{t}$ defined on $p$-groups with $p$ odd, we recover the earlier obstruction group computations done by Bouc and Thévenaz [6]. We also recover the obstruction group calculations done by Coşkun [8] for the dual of the rational representation ring functor $R_{\mathbb{Q}}^{*}$ and for the Borel-Smith functor $C_{b}$ (Propositions 7.2 and 7.6). We also calculate the obstruction group $\operatorname{Obs}\left(D_{t}(G)\right.$ ) for a 2-group $G$ (see Proposition 7.3).

In Section 7 we consider the obstruction group for the Dade group functor $D$ defined over $p$-groups with $p$ odd. We first prove a vanishing result $H^{1}\left(\mathcal{D}_{G}^{*} ; B^{*}\right)=0$ for the dual of the Burnside ring functor $B^{*}$ using a base change spectral sequence for ext-groups (see Lemma 7.5). As a consequence of this vanishing result we prove the following theorem. 
Theorem 1.5. Let $G$ be a $p$-group with $p$ odd. Let $D$ denote the $p$-biset functor for the Dade group. Then there is an exact sequence of abelian groups

$$
0 \rightarrow \operatorname{Obs}(D(G)) \rightarrow H^{1}\left(\mathcal{A}_{\geq 2}(G) / G ; \mathbb{Z}\right) \rightarrow H^{1}\left(\mathcal{A}_{\geq 2}(G) / G ; \mathbb{Z} / 2\right)
$$

where the second map is induced by the $\bmod 2$ reduction $\operatorname{map} \mathbb{Z} \rightarrow \mathbb{Z} / 2$.

This theorem is proved as Theorem 7.9 in Section 7. One of the key ingredients is the computation of $H^{1}\left(\mathcal{D}_{G}^{*} ; F\right)$ for a rhetorical $p$-biset functor satisfying certain properties (see Proposition 7.8).

The paper is organized as follows: We introduce the destriction algebra and the orbit category of sections in Section 2. The obstruction groups for the gluing problem are defined in Section 3. Theorem 1.3 is also proved in this section. In Section 4 we define the reduced cohomology groups for the category $\mathcal{D}_{G}^{\mathcal{C}}$, and apply a theorem of Jackowski and Słominska to these reduced cohomology groups. In Section 5 we show that the reduced cohomology groups of $\mathcal{D}_{G}^{\mathcal{C}}$ can be calculated as the cohomology groups of the Quillen category $\mathcal{A}_{p}(G)$ when $G$ is a $p$-group (Theorem 1.4). In Section 6 we prove Theorem 6.8, which gives an explicit description of the obstruction group $\operatorname{Obs}(F(G))$ for a rhetorical $p$-biset functor $F$. Finally in Section 7 we apply Theorem 6.8 to calculate the obstruction groups for some rhetorical $p$-biset functors related to endo-permutation modules. Theorem 1.5 is proved in this final section.

Acknowledgments: The second author is supported by a Tübitak 1001 project (grant no: $116 \mathrm{~F} 194)$. We thank the referee for careful reading of the paper and for many helpful suggestions that improved the paper.

\section{Destriction algebra and the orbit category of sections}

In this section, we introduce basic definitions about biset functors and destriction algebra. Then we define the orbit category of sections $\mathcal{D}_{G}^{\mathcal{C}}$ over a collection of sections $\mathcal{C}$ and prove that the module category over the orbit category of sections is equivalent to the module category over the destriction algebra.

\subsection{Biset functors}

Let $K$ and $H$ be finite groups. A $(K, H)$-biset is a finite set $X$ together with a left $K$-action and a right $H$-action such that $k(x h)=(k x) h$ for every $k \in K, h \in H$, and $x \in X$. A $(K, H)$-biset $X$ can be considered as a left $K \times H$-set with the action given by $(k, h) x=k x h^{-1}$. We define the Burnside group of $(K, H)$-bisets $B(K, H)$ as the Burnside group $B(K \times H)$ of left $K \times H$-sets. Given another finite group $L$, we define a composition product

$$
-\times_{H}-: B(K, H) \times B(H, L) \rightarrow B(K, L)
$$


as the linear extension of the correspondence $(X, Y) \mapsto X \times_{H} Y$ where $X \times_{H} Y=$ $(X \times Y) / H$ is the set of $H$-orbits of the set $X \times Y$ under the action $h \cdot(x, y):=\left(x h^{-1}, h y\right)$.

Let $R$ be a commutative ring with unity. The $R$-biset category $R \mathcal{B}$ is a category whose objects are all finite groups, and in which the morphisms from the finite group $H$ to the finite group $K$ are given by the Burnside module $R \otimes_{\mathbb{Z}} B(K, H)$ of $(K, H)$-bisets. The composition in the biset category is given by the above composition product. For any finite group $H$, the identity morphism of $H$ in $R \mathcal{B}$ is equal to $R \otimes \mathrm{id}_{H}$, where $\mathrm{id}_{H}={ }_{H} H_{H}$ is the identity biset at $H$.

Recall that if $U, V$ are subgroups of a finite group $G$ such that $V \unlhd U$, then the quotient group $U / V$ is called a subquotient of $G$, and the pair $(U, V)$ is called a section of the group $G$. Let $\mathcal{X}$ be a finite set of finite groups closed under taking subquotients up to isomorphism. By this we mean that if $G \in \mathcal{X}$, then a subquotient $U / V$ of $G$ is isomorphic to a group in $\mathcal{X}$.

Definition 2.1. The $R$-biset category $R \mathcal{B}^{\mathcal{X}}$ over the collection $\mathcal{X}$ is the full subcategory of the biset category $R \mathcal{B}$ whose objects are groups in $\mathcal{X}$. An $R$-linear functor

$$
F: R \mathcal{B}^{\mathcal{X}} \rightarrow R \text {-mod }
$$

is called a biset functor defined on the collection $\mathcal{X}$ over $R$.

To define a biset functor for a fixed finite group $G$, we take $\mathcal{X}$ as the collection $\mathcal{X}^{s}$ which is defined as the set of all finite groups indexed by sections $(U, V)$ of $G$, where the group in $\mathcal{X}^{s}$ corresponding to the section $(U, V)$ is the quotient group $U / V$. In this case we denote the corresponding $R$-biset category by $R \mathcal{B}_{G}$, and an $R$-linear functor $F: R \mathcal{B}_{G} \rightarrow R$-mod is called a biset functor for $G$ over $R$.

We also recall the definition of the alchemic algebra for an arbitrary collection $\mathcal{X}$.

Definition 2.2. The alchemic algebra for $\mathcal{X}$ over $R$ is the $R$-algebra

$$
R \Gamma^{\mathcal{X}}=\bigoplus_{K, H \in \mathcal{X}} R B(K, H)
$$

with multiplication given by biset composition products. A biset functor $F$ defined on $\mathcal{X}$ over $R$ is defined to be an $R \Gamma^{\mathcal{X}}$-module (see [1] for details).

It is shown in [1, pg. 3816] that this definition of a biset functor as an $R \Gamma^{\mathcal{X}}$-module coincides with the definition given in Definition 2.1.

By [3, Lemma 2.3.26], any transitive $(K, H)$-biset is a composition of five elementary bisets. To introduce our notation, let $H \leq G$ and $N \unlhd G$ be subgroups of $G$ and $\phi: G^{\prime} \rightarrow G$ be an isomorphism. Then the set $G$ can be regarded as a $(G, H)$-biset, as an $(H, G)$-biset or as a $\left(G, G^{\prime}\right)$-biset via the usual actions. These bisets are called the induction biset $\operatorname{Ind}_{H}^{G}$, the restriction biset $\operatorname{Res}_{H}^{G}$ and the isogation biset $\mathrm{Iso}_{G, G^{\prime}}^{\phi}$, respectively. In addition, 
the set $G / N$ can be regarded as a $(G, G / N)$-biset or as a $(G / N, G)$-biset. These are called the inflation biset $\operatorname{Inf}_{G / N}^{G}$ and deflation biset $\operatorname{Def}_{G / N}^{G}$. With this notation, for a subgroup $J \leq K \times H$, we have

$$
\left(\frac{K \times H}{J}\right)=\operatorname{Ind}_{P}^{K} \operatorname{Inf}_{P / A}^{P} \operatorname{Iso}_{P / A, Q / B}^{\lambda} \operatorname{Def}_{Q / B}^{Q} \operatorname{Res}_{Q}^{H}
$$

where $P=\{k \in K \mid \exists h \in H:(k, h) \in J\}$ and $A=\{k \in K \mid(k, 1) \in J\}$. The subgroups $Q$ and $B$ are defined in a similar way. The isomorphism $\lambda: Q / B \rightarrow P / A$ is the one induced by $J$.

In particular, the alchemic algebra for $\mathcal{X}$ over $R$ is generated by its elementary bisets. An $R$-free basis for $R \Gamma^{\mathcal{X}}$ can be found in [17, Lemma 2.2].

\subsection{Destriction algebra}

Let $R$ be a commutative ring with unity and $G$ be a finite group. Let $\mathcal{X}^{s}$ denote a collection of finite groups defined in the previous section. For a section $(U, V)$ of $G$, the composition of the deflation map $\operatorname{Def}_{U / V}^{U}$ and the restriction map $\operatorname{Res}_{U}^{G}$ is called the destriction biset and is denoted by $\operatorname{Defres}_{U / V}^{G}$ or shortly by $\operatorname{Des}_{U / V}^{G}$. For a section $(M, L)$ of $G$, if $(U, V)$ is another section of $G$ with $L \leq V \leq U \leq M$, then there is a $(U / V, M / L)$-biset given by

$$
\operatorname{Iso}_{U / V,(U / L) /(V / L)}^{\lambda} \circ \operatorname{Def}_{(U / L) /(V / L)}^{U / L} \circ \operatorname{Res}_{U / L}^{M / L}
$$

where $\lambda$ is the canonical isomorphism. With an abuse of notation, we denote this composition by $\operatorname{Des}_{U / V}^{M / L}$, and call it the destriction $(U / V, M / L)$-biset.

Definition 2.3. Let $R, G$ and $\mathcal{X}^{s}$ be as above.

(1) The $R$-destriction category $R \mathcal{D E} \mathcal{S}_{G}$ is the subcategory of the $R$-biset category $R \mathcal{B}_{G}$ with objects the groups in $\mathcal{X}^{s}$, in which morphisms from $H=M / L$ to $K=U / V$ are given by the bisets of the form

$$
\mathrm{c}_{K, K^{g}}^{g} \operatorname{Des}_{K^{g}}^{H}
$$

where $(U, V)^{g} \preceq(M, L)$ and $g \in G$.

(2) The native destriction algebra $\nabla_{G}^{\prime}(R)$ is defined as the subalgebra of the alchemic algebra $R \Gamma^{\mathcal{X}^{s}}$ generated by the bisets of the form $c_{g_{S, S}}^{g}=\operatorname{Iso}_{g_{S, S}}^{g}$ and $\operatorname{Des}_{S}^{T}$ where $g \in G$ and $S=U / V$ and $T=M / L$ with $(U, V) \preceq(M, L)$.

Note that it follows from [17, Lemma 2.2] that the set

$$
\left\{\mathrm{c}_{K, K^{g}}^{g} \operatorname{Des}_{K^{g}}^{H} \mid K=U / V, H=M / L \text { with }(U, V)^{g} \preceq(M, L), g \in U \backslash G\right\}
$$


is an $R$-free basis for $\nabla_{G}(R)$. Also as in the case of biset functors and the alchemic algebra, the category of modules over $\nabla_{G}^{\prime}(R)$ can be identified by the category of $R$-linear functors $R \mathcal{D E S} \mathcal{S}_{G} \rightarrow R$-mod. There is a restriction functor from the category of biset functors to the category of native destriction functors, induced by the inclusion map $\nabla_{G}^{\prime}(R) \hookrightarrow R \Gamma^{\mathcal{X}^{s}}$ of the subalgebra.

Now we define an abstract destriction algebra with generators and relations that will be more useful for our purposes.

Definition 2.4. Let $R, G$ and $\mathcal{X}^{s}$ be as above.

(1) The destriction algebra $\nabla_{G}(R)$ is the algebra generated by the symbols of the form (G1) $\mathrm{c}_{g_{K, K}}^{g}$ for each $g \in G$ and $K=U / V$ in $\mathcal{X}^{s}$, called a conjugation and

(G2) $\operatorname{Des}_{K}^{H}$ for each pair $K=U / V$ and $H=M / L$ in $\mathcal{X}^{s}$ such that $(U, V) \preceq(M, L)$, called a destriction (in this case we also write $K \preceq H$ )

subject to the following relations

(R1) $\mathrm{c}_{H, H}^{h}=\operatorname{Des}_{H}^{H}$ for any $H=M / L$ and $h \in M$,

(R2) $\mathrm{c}_{g g^{\prime} H, g^{\prime} H}^{g} \mathrm{c}_{g^{\prime} H, H}^{g^{\prime}}=\mathrm{c}_{g g^{\prime} H, H}^{g g^{\prime}}$ and $\operatorname{Des}_{L}^{K} \operatorname{Des}_{K}^{H}=\operatorname{Des}_{L}^{H}$ for any $g, g^{\prime} \in G$ and $L \preceq$ $K \preceq H$,

(R3) $\operatorname{Des}_{K}^{H} \mathrm{c}_{H, H^{g}}^{g}=\mathrm{c}_{K, K^{g}}^{g} \operatorname{Des}_{K^{g}}^{H^{g}}$ for any $g \in G$ and $K \preceq H$, and

(R4) $1=\sum_{U / V \in \mathcal{X}^{s}} \mathrm{c}_{U / V}$ where $\mathrm{c}_{U / V}=\mathrm{c}_{U / V, U / V}^{1}$ is the conjugation associated to the identity element 1 of $G$.

(2) A destriction functor for $G$ over $R$ is defined as a module over the destriction algebra $\nabla_{G}(R)$.

It is straightforward to show, using arguments in [17, Lemma 2.2], that the destriction algebra has a basis consisting of symbols of the form $\mathrm{c}_{K, K^{g}}^{g} \operatorname{Des}_{K^{g}}^{H}$. Hence there is a homomorphism of algebras $\varphi: \nabla_{G}(R) \rightarrow R \Gamma^{\mathcal{X}^{s}}$ defined by mapping the symbol $\mathrm{c}_{K, K^{g}}^{g} \operatorname{Des}_{K^{g}}^{H}$ to the biset $\mathrm{c}_{K, K^{g}}^{g} \operatorname{Des}_{K^{g}}^{H}$. By definition the image of the homomorphism $\varphi$ is the native destriction algebra $\nabla_{G}^{\prime}(R)$. The homomorphism $\varphi$ is not injective in general since some conjugations may induce isomorphic bisets, for example, if $g$ centralizes $K$, then $\mathrm{c}_{K, K^{g}}^{g}=\mathrm{c}_{K, K}^{1}$ as bisets. Every biset functor $F$ can be regarded as a destriction functor for $G$ over $R$ via the homomorphism $\varphi: \nabla_{G}(R) \rightarrow R \Gamma^{\mathcal{X}^{s}}$.

In the next section, we define a small category and express destriction functors as functors from this small category to the category of $R$-modules.

\subsection{The orbit category of sections}

Let $G$ be a finite group. We call a set of sections of $G$ a collection of sections of $G$ if it is closed under conjugation. Let $\mathcal{C}$ be a collection of sections of a finite group $G$. The orbit category of sections $\mathcal{D}_{G}^{\mathcal{C}}$ over the collection $\mathcal{C}$ is defined as in Definition 1.2. When $\mathcal{C}$ is the collection of all sections of $G$, we denote the category of sections with $\mathcal{D}_{G}$. Note that the category $\mathcal{D}_{G}^{\mathcal{C}}$ is an EI-category (all endomorphisms are isomorphisms) and 
we have the isomorphism $\operatorname{Aut}_{\mathcal{D}_{G}}((U, V)) \cong N_{G}(U, V) / U$ of groups where $N_{G}(U, V)=$ $N_{G}(U) \cap N_{G}(V)$.

Remark 2.5. Recall that the orbit category $\mathcal{O}_{G}^{\mathcal{C}}$ of a finite group $G$, over a collection $\mathcal{C}$ of subgroups of $G$, is the category whose objects are the subgroups of $G$ and whose morphisms from $H$ to $K$ are the set of $G$-maps $G / H \rightarrow G / K$, with a composition given by the composition of functions. We can identify the set of morphisms from $H$ to $K$ with right cosets $K g$ with $g \in G$ satisfying $g H^{-1} \leq K$. Note that given a collection of subgroups $\mathcal{C}$, if we define a collection of sections $\mathcal{C}^{\prime}$ as a collection formed by sections $S=(U, V)$ with $V=1$ and $U \in \mathcal{C}$, then the category of sections $\mathcal{D}_{G}^{\mathcal{C}^{\prime}}$ is isomorphic to the orbit category $\mathcal{O}_{G}^{\mathcal{C}}$. The notion of orbit category of sections is a generalization of the orbit category of a group.

The category $\mathcal{D}_{G}$ is closely connected to the destriction algebra $\nabla_{G}(R)$ introduced in Section 2.2.

Proposition 2.6. Let $\mathcal{C}$ be a collection of sections closed under taking subsections. Let $\overline{\mathcal{D}_{G}^{\mathcal{C}}}$ denote the opposite category of $\mathcal{D}_{G}^{\mathcal{C}}$, and let $1_{\nabla}^{\mathcal{C}}$ denote the sum $\sum_{(U, V) \in \mathcal{C}} \mathrm{c}_{U / V}$ in the destriction algebra $\nabla_{G}(R)$. Set $\nabla_{G}^{\mathcal{C}}(R)=1_{\nabla}^{\mathcal{C}} \nabla_{G}(R) 1_{\nabla}^{\mathcal{C}}$. Then the map

$$
j_{G}: R \overline{\mathcal{D}_{G}^{\mathcal{C}}} \rightarrow \nabla_{G}^{\mathcal{C}}(R)
$$

that takes the morphism $M g$ from $(U, V)$ to $(M, L)$ in $\mathcal{D}_{G}^{\mathcal{C}}$ to the basis element $\mathrm{c}_{S,{ }_{S}}^{g^{-1}} \operatorname{Des}_{{ }_{S}}^{T}$ in $\nabla_{G}^{\mathcal{C}}(R)$, where $S=U / V$ and $T=M / L$, is an isomorphism of $R$-algebras.

Proof. The map $j_{G}$ is well-defined since, given $x \in M$, we have

$$
\mathrm{c}_{S,{ }^{x g} S}^{(x g)^{-1}} \operatorname{Des}_{x g}^{T}{ }^{T}=\mathrm{c}_{S,{ }_{S}}^{g^{-1}} \mathrm{c}_{g_{S},{ }^{x g}{ }_{S}}^{x^{-1}} \operatorname{Des}_{x g}^{T}{ }^{T}=\mathrm{c}_{S,{ }_{S}}^{g^{-1}} \operatorname{Des}_{g_{S}}^{T^{x}} \mathrm{c}_{T^{x}, T}^{x^{-1}}=\mathrm{c}_{S,{ }_{S}}^{g^{-1}} \operatorname{Des}_{g_{S}}^{T}
$$

by the composition product formula for bisets. Also, by the transitivity of destriction and conjugation, $j_{G}$ preserves products. Therefore $j_{G}$ is an algebra map, which is clearly surjective. To prove that it is also injective, let $c_{S,{ }_{S}}^{g^{-1}} \operatorname{Des}_{g_{S}}^{T}=\mathrm{c}_{S, h}^{h^{-1}} \operatorname{Des}_{h}^{T}$ for $M g, M h \in$ $\operatorname{Hom}_{\overline{\mathcal{D}}_{G}}(T, S)$. This equality holds if there exists $x \in M$ such that ${ }^{x g} S={ }^{h} S$ and

$$
\mathrm{c}_{S,{ }^{g} S}^{g^{-1}}=\mathrm{c}_{S,{ }^{h} S^{-1}}^{h^{-1}} \mathrm{c}_{x g}^{x},_{, S} .
$$

In particular we have $g h^{-1} x \in{ }^{g} U \leq M$. Since $x \in M$, we get $g h^{-1} \in M$, that is, $M g=M h$, as required.

\subsection{Modules over destriction algebra}

Given a biset functor or a destriction functor $F$, there is an associated contravariant functor 


$$
F: \mathcal{D}_{G}^{\mathcal{C}} \rightarrow R \text {-mod, }
$$

defined as follows: We first consider the biset functor $F$ as an $R \overline{\mathcal{D}_{G}^{\mathcal{C}}}$-module via the composition

$$
R \overline{\mathcal{D}_{G}^{\mathcal{C}}} \stackrel{j_{G}}{\longrightarrow} \nabla_{G}^{\mathcal{C}}(R) \stackrel{\varphi}{\longrightarrow} R \Gamma^{\mathcal{C}}
$$

where the isomorphism $j_{G}$ is given in Proposition 2.6. Then we use the correspondence between the contravariant functors $\mathcal{D}_{G}^{\mathcal{C}} \rightarrow R$-mod and the modules over the category algebra $R \overline{\mathcal{D}_{G}^{\mathcal{C}}}$ (see [14, Thm 7.1]). Because of this correspondence contravariant functors $\mathcal{D}_{G}^{\mathcal{C}} \rightarrow R$-mod are usually called (right) $R \mathcal{D}_{G}^{\mathcal{C}}$-modules. Conversely, every contravariant functor $F: \mathcal{D}_{G}^{\mathcal{C}} \rightarrow R$-mod can be considered as a $\nabla_{G}^{\mathcal{C}}(R)$-module (destriction functor) via the isomorphism $j_{G}$.

Note that under these identifications, the category algebra of the orbit category $\mathcal{O}_{G}^{\mathcal{C}}$ over a collection $\mathcal{C}$ of subgroups of $G$ is identified with the algebra

$$
\rho_{G}:=\nabla_{G}^{\mathcal{C}^{\prime}}(R)=1_{\nabla}^{\mathcal{C}^{\prime}} \nabla_{G}(R) 1_{\nabla}^{\mathcal{C}^{\prime}}
$$

where $\mathcal{C}^{\prime}=\{(H, 1) \mid H \in \mathcal{C}\}$. The algebra $\rho_{G}$ is called the restriction algebra for $G$ over $R$. The representation theory of $\rho_{G}$ is well-known. Moreover representation theory of $\nabla_{G}^{\mathcal{C}}(R)$ for an arbitrary collection $\mathcal{C}$ is very similar to that of the restriction algebra. Simple and projective modules can be described using the following constructions. Alternatively, one can use the general results about the representations of EI-categories to construct these modules.

Let $\nabla:=\nabla_{G}^{\mathcal{C}}(R)$. There is a subalgebra $\Omega$ of $\nabla$, called the conjugation subalgebra, generated by all conjugations in $\nabla$. It is isomorphic to the quotient of $\nabla$ by the ideal generated by all proper destriction maps, that is by all $\operatorname{Des}_{K}^{H}$ with $H \neq K$. This means that there are several functors between the categories of modules of these algebras, namely there are induction, coinduction and inflation from $\Omega$-mod to $\nabla$-mod and deflation, codeflation and restriction from $\nabla$-mod to $\Omega$-mod. Two of these functors are easy to identify. The restriction functor $\operatorname{Res}_{\Omega}^{\nabla}$ from $\nabla$-modules to $\Omega$-modules is the usual restriction of the $\nabla$-action. The inflation functor $\operatorname{Inf}_{\Omega}^{\nabla}$ is the restriction functor along the canonical epimorphism $\nabla \rightarrow \Omega$ of algebras.

First we describe the $\Omega$-modules. Given a section $H=(U, V)$ of $G$, we write $\mathrm{c}_{[H]}$ for the sum of all idempotents $\mathrm{c}_{M / L}$ over all sections $(M, L)$ which are $G$-conjugate to $(U, V)$. Then $\Omega_{[H]}=\mathrm{c}_{[H]} \Omega \mathrm{c}_{[H]}$ is a two-sided ideal of $\Omega$ and

$$
\Omega=\bigoplus_{H \preceq G G} \Omega_{[H]} .
$$

In particular, any $\Omega$-module $F$ can be decomposed as $F=\oplus_{H \preceq{ }_{G} G} F_{[H]}$ where $F_{[H]}=$ $\mathrm{c}_{[H]} F$. 
Given a section $H$ of $G$, the algebra $\Omega_{H}:=\mathrm{c}_{H} \Omega \mathrm{c}_{H}=\mathrm{c}_{H} \Omega_{[H]} \mathrm{c}_{H}$ is isomorphic to the group algebra $R\left[N_{G}(U, V) / U\right]$ and is also a (non-unital) subalgebra of $\Omega_{[H]}$. Hence there is an obvious restriction functor $\operatorname{Res}_{\Omega_{H}}^{\Omega_{[H]}}: \Omega_{[H]}$-mod $\rightarrow \Omega_{H}$-mod given on objects by $\mathrm{c}_{H} \Omega_{[H]} \otimes_{\Omega_{[H]}}$-. Its left adjoint is the induction functor $\operatorname{Ind}_{\Omega_{H}}^{\Omega_{[H]}}=\Omega_{[H]} \mathrm{c}_{H} \otimes_{\Omega_{H}}-$. It is easy to show that there are natural equivalences

$$
\operatorname{Ind}_{\Omega_{H}}^{\Omega_{[H]}} \circ \operatorname{Res}_{\Omega_{H}}^{\Omega_{[H]}} \cong \operatorname{Id}_{\Omega_{[H]}-\bmod } \text { and } \operatorname{Res}_{\Omega_{H}}^{\Omega_{[H]}} \circ \operatorname{Ind}_{\Omega_{H}}^{\Omega_{[H]}} \cong \operatorname{Id}_{\Omega_{H}-\bmod }
$$

In particular the algebra $\Omega_{[H]}$ is Morita equivalent to the group algebra $\Omega_{H}$.

Now it is straightforward to see that the algebra $\Omega$ is Morita equivalent to the product algebra $\prod_{H \preceq G G} \Omega_{H}$. Hence the representations of $\Omega$ are just tuples of group representations for the various groups appearing in the product. In particular, the simple $\Omega$-modules are the functors $S_{H, I}^{\Omega}$ where $H \preceq G$ and $I$ is a simple $\Omega_{H}$-module such that $\mathrm{c}_{[K]} S_{H, I}^{\Omega}=0$ if $K$ is not conjugate to $H$ in $G$ and $S_{H, I}^{\Omega}(H)=I$. We call such a functor which has a support only on a unique conjugacy class of sections an atomic functor. Similarly, if $P_{I}$ is a projective cover of $I$ as an $\Omega_{H}$-module, then the atomic functor $P_{H, I}^{\Omega}$ with value $P_{I}$ at $H$ is a projective cover of $S_{H, I}^{\Omega}$. We also have

$$
S_{H, I}^{\Omega}=\operatorname{Ind}_{\Omega_{H}}^{\Omega} I \quad \text { and } \quad P_{H, I}^{\Omega}=\operatorname{Ind}_{\Omega_{H}}^{\Omega} P_{I} .
$$

With this notation we have the following description of simple and projective indecomposable $\nabla$-modules. Note that although the classification of simple and projective modules for $\nabla$ are similar to that of biset functors as in [3], the following result does not follow from the later classification. In [3], the categories of bisets always contains all inductions and restrictions hence $\nabla$ is not an example of these general results.

Proposition 2.7. Let $H \preceq G$ be a section of $G$, let $I$ be a simple $\Omega_{H}$-module and $P_{I}$ be its projective cover. The following statements hold.

(1) The $\nabla$-module $S_{H, I}^{\nabla}=\operatorname{Inf}_{\Omega}^{\nabla} S_{H, I}^{\Omega}$ is simple. Moreover, any simple $\nabla$-module is of this form for some pair $(H, I)$ with $H \preceq G$ and $I$ a simple $\Omega_{H}$-module.

(2) The $\nabla$-module $P_{H, I}^{\nabla}=\operatorname{Ind}_{\Omega}^{\nabla} P_{H, I}^{\Omega}$ is projective indecomposable with simple head $S_{H, I}^{\nabla}$.

Proof. It is clear that the $\nabla$-module $S_{H, I}^{\nabla}$ is simple. To see that any simple $\nabla$-module is of this form, note that for any $\nabla$-module $F$ and any sections $(U, V)$ and $(M, L)$ of $G$ with $F(U / V) \neq 0 \neq F(M / L)$ such that $|U / V| \geq|M / L|$ and $(U, V) \neq_{G}(M, L)$, the $R$-module $F(M / L)$ generates a proper non-zero $\nabla$-submodule of $F$. Thus a simple $\nabla$-module must be atomic. Moreover if $S$ is a simple $\nabla$-module with $S(H) \neq 0$, then clearly $S(H)$ is simple.

To prove the second part, write

$$
P_{H, I}^{\nabla}=\operatorname{Ind}_{\Omega}^{\nabla} P_{H, I}^{\Omega}=\operatorname{Ind}_{\Omega}^{\nabla} \operatorname{Ind}_{\Omega_{H}}^{\Omega} P_{I}=\operatorname{Ind}_{\Omega_{H}}^{\nabla} P_{I}=\nabla \otimes_{\Omega_{H}} P_{I}
$$


Since $\nabla$ is a free right $\Omega_{H}$-module, by general properties of tensor product, the $\nabla$-module $P_{H, I}^{\nabla}$ is projective and it is indecomposable since we have the following isomorphisms of rings.

$\operatorname{End}_{\nabla}\left(P_{H, I}^{\nabla}\right)=\operatorname{Hom}_{\nabla}\left(\nabla \otimes_{\Omega_{H}} P_{I}, \nabla \otimes_{\Omega_{H}} P_{I}\right) \cong \operatorname{Hom}_{\Omega_{H}}\left(P_{I}, \nabla \otimes_{\Omega_{H}} P_{I}\right) \cong \operatorname{Hom}_{\Omega_{H}}\left(P_{I}, P_{I}\right)$

Here the last isomorphism holds since as $\Omega_{H}$-modules, there is an isomorphism

$$
\nabla \otimes_{\Omega_{H}} P_{I}=\mathrm{c}_{H} \nabla \mathrm{c}_{H} \otimes_{\Omega_{H}} P_{I} \cong \Omega_{H} \otimes_{\Omega_{H}} P_{I} \cong P_{I}
$$

Since by definition, $P_{I}$ is indecomposable, the $\operatorname{ring} \operatorname{End}_{\nabla}\left(P_{H, I}^{\nabla}\right)$ is local, and hence $P_{H, I}^{\nabla}$ is indecomposable.

Corollary 2.8. Let $H \preceq G$ and $I$ be a simple $\Omega_{H}$-module. Then for any section $K \preceq G$, there is an isomorphism

$$
P_{H, I}^{\nabla}(K) \cong R \operatorname{Hom}_{\nabla}(H, K) \otimes_{\Omega_{H}} P_{I}
$$

of $\Omega_{K}$-modules.

This gives a characterization of projective indecomposable $R \mathcal{D}_{G}^{\mathcal{C}}$-modules via the isomorphism between $R \overline{\mathcal{D}}_{G}^{\mathcal{C}}$ and $\nabla_{G}^{\mathcal{C}}(R)$. Alternatively we can define projective $R \mathcal{D}_{G}^{\mathcal{C}}$-modules as direct summands of free $R \mathcal{D}_{G}^{\mathcal{C}}$-modules, which are defined as follows:

Definition 2.9. For any section $(U, V)$ of $G$, let $P_{(U, V)}$ denote the $R \mathcal{D}_{G}^{\mathcal{C}}$-module

$$
P_{(U, V)}(-):=R \operatorname{Hom}_{\mathcal{D}_{G}}(-,(U, V))
$$

with destriction and conjugation maps defined by composition.

By the Yoneda lemma these modules satisfy the property that if $(U, V) \in \mathcal{C}$, then for every $R \mathcal{D}_{G}^{\mathcal{C}}$-module $F$,

$$
\operatorname{Hom}_{R \mathcal{D}_{G}^{\mathcal{C}}}\left(P_{(U, V)}, F\right) \cong F(U / V)
$$

This gives, in particular, that $P_{(U, V)}$ is a projective $R D_{G}^{\mathcal{C}}$-module. Note that this definition works more generally for any EI-category. We will be using these definitions in the next section when we are writing projective resolutions for $R \mathcal{D}_{G}^{\mathcal{C}}$-modules.

Remark 2.10. Note that to simplify the notation we write $F(U / V)$ for $F((U, V))$ when $F$ is an $R \mathcal{D}_{G}$-module. We also write $F(G)$ for $F(G / 1)$ to simplify the notation further. 


\section{Obstruction groups for the gluing problem}

In this section we introduce an obstruction theory for the gluing problem stated in Definition 1.1. Let $G$ be a finite group, and let $\mathcal{D}_{G}$ denote the orbit category of sections in $G$. Let $\underline{R}$ denote the constant functor, that is, the $R \mathcal{D}_{G}$-module with values isomorphic to the trivial module $R$ at every section $(U, V)$, where all the destriction and conjugation maps are the identity maps.

Lemma 3.1. The constant functor $\underline{R}$ is isomorphic to $P_{(G, 1)}$. In particular, $\underline{R}$ is a projective $R \mathcal{D}_{G}$-module, and there is an isomorphism

$$
\operatorname{Hom}_{R \mathcal{D}_{G}}(\underline{R}, F) \cong F(G)
$$

for every $R \mathcal{D}_{G}$-module $F$.

Proof. For every $(U, V) \in \mathcal{D}_{G}$, there is a unique morphism $f_{(U, V)} \in \operatorname{Hom}_{\mathcal{D}_{G}}((U, V),(G, 1))$, which is the coset $G \cdot 1$, and these morphisms are mapped to each other under morphisms in $\mathcal{D}_{G}$, meaning that, if $f:(U, V) \rightarrow(M, L)$ is a morphism in $\mathcal{D}_{G}$, then

$$
f^{*}\left(f_{(M, L)}\right)=f_{(M, L)} \circ f=f_{(U, V)},
$$

where $f^{*}$ denotes the morphism induced by $f$ in $P_{(G, 1)}$. This gives the isomorphism $\underline{R} \cong P_{(G, 1)}$. The second part follows from the properties of modules $P_{(U, V)}$ that were explained in Section 2.4.

Let $\mathcal{C}$ be a collection of sections of $G$. In the rest of the section we assume that $\mathcal{C}$ is closed under taking subsections, i.e., if $(M, L) \in \mathcal{C}$ and $(U, V) \preceq(M, L)$, then $(U, V) \in \mathcal{C}$. Let $J^{\mathcal{C}}$ denote the subfunctor of $\underline{R}$ that has the value $R$ on the sections $(U, V)$ in $\mathcal{C}$, and is equal to zero on the other sections. Let $T^{\mathcal{C}}$ denote the quotient module $\underline{R} / J^{\mathcal{C}}$. Note that for any $R \mathcal{D}_{G}$-module $F$, we have

$$
\operatorname{Hom}_{R \mathcal{D}_{G}}\left(J^{\mathcal{C}}, F\right) \cong \lim _{(U, V) \in \mathcal{D}_{G}^{\mathcal{C}}} F(U / V)
$$

The short exact sequence $0 \rightarrow J^{\mathcal{C}} \rightarrow \underline{R} \rightarrow T^{\mathcal{C}} \rightarrow 0$ gives a 4 -term exact sequence that takes the following form when we apply the isomorphisms above:

$$
0 \longrightarrow \operatorname{Hom}_{R \mathcal{D}_{G}}\left(T^{\mathcal{C}}, F\right) \longrightarrow F(G) \stackrel{r_{G}^{F}}{\longrightarrow} \lim _{(U, \overleftarrow{V}) \in \mathcal{D}_{G}^{\mathcal{C}}} F(U / V) \longrightarrow \operatorname{Ext}_{R \mathcal{D}_{G}}^{1}\left(T^{\mathcal{C}}, F\right) \longrightarrow 0
$$

The map $r_{G}^{F}$ induced by the inclusion $J^{\mathcal{C}} \rightarrow \underline{R}$ coincides with the detection map defined by

$$
r_{G}^{F}(f)=\left(\operatorname{Defres}_{U / V}^{G} f\right)_{(U, V) \in \mathcal{D}_{G}^{\mathcal{C}}}
$$


This 4-term exact sequence can be considered as the solution for the gluing problem for a collection $\mathcal{C}$. The obstruction group is given by

$$
\operatorname{Obs}^{\mathcal{C}}(F(G)) \cong \operatorname{Ext}_{R \mathcal{D}_{G}}^{1}\left(T^{\mathcal{C}}, F\right)
$$

and the uniqueness is determined by the group $\operatorname{Hom}_{R \mathcal{D}_{G}}\left(T^{\mathcal{C}}, F\right)$.

Remark 3.2. Gluing problems for more general collections of sections are considered in $[6$, Section 4]. One of these collections is the collection $\mathcal{C}$ of all sections $(U, V)$ where the quotient group $U / V$ is an elementary abelian $p$-group. In the case where $G$ is a $p$-group with $p$ odd, it is shown in [6, Corollary 4.3] that the detection map $r_{G}^{F}$ for this collection is an isomorphism for the biset functor $D_{t}$ of the torsion part of the Dade group. Another collection of sections considered in [6] is the collection of the sections $\left(N_{G}(Q), Q\right)$ where $Q$ is a $p$-centric subgroup of $G$. Note that in this case, the family of sections is not closed under taking subsections. We discuss the gluing problem for such collections in the next section.

We now focus on the case where $\mathcal{C}$ is the collection of all sections $(U, V)$ of $G$ such that $V \neq 1$. In this case we denote the $R \mathcal{D}_{G^{-m o d u l e s}} T^{\mathcal{C}}$ and $J^{\mathcal{C}}$ simply by $T$ and $J$.

Proposition 3.3. Let $F$ be a destriction functor or a biset functor for $G$ over $R$. Then the map

$$
\phi: \operatorname{Hom}_{R \mathcal{D}_{G}}(J, F) \rightarrow \lim _{1<\overleftarrow{H \leq G}} F\left(N_{G}(H) / H\right)
$$

defined by $\phi(f)=\left(f\left(1_{N_{G}(H) / H}\right)\right)_{1<H \leq G}$ is an isomorphism of $R$-modules.

Proof. It is clear that the map $\phi$ is additive. To prove that it is well-defined, we need to show that $\phi(f)$ is a gluing data for every $f \in \operatorname{Hom}_{R \mathcal{D}_{G}}(J, F)$, i.e., we need to verify that $\phi(f)$ satisfies the conjugation and destriction invariance conditions given in Definition 1.1. The conjugation invariance of $\phi(f)$ is trivial and we leave the justification to the reader. To prove the destriction invariance, note that for every $K \unlhd H \leq G$ we have

$$
\operatorname{Des}_{N_{G}(H, K) / H}^{N_{G}(H) / H} 1_{N_{G}(H) / H}=1_{N_{G}(H, K) / H}=\operatorname{Des}_{N_{G}(H, K) / H}^{N_{G}(K) / K} 1_{N_{G}(K) / K},
$$

and hence the destriction invariance follows from the fact that $f$ is an $R \mathcal{D}_{G}$-module homomorphism.

Now let

$$
\psi: \lim _{1<\overleftarrow{H \leq G}} F\left(N_{G}(H) / H\right) \rightarrow \operatorname{Hom}_{R \mathcal{D}_{G}}(J, F)
$$

be the map defined by $\psi\left(\left(f_{H}\right)_{1<H \leq G}\right)=\left(1_{K / L} \mapsto \operatorname{Res}_{K / L}^{N_{G}(L) / L} f_{L}\right)$. This map is additive since the restriction map is additive. Moreover it is clear that $\phi$ and $\psi$ are inverse to each 
other. Therefore it remains to check that the map $\psi$ is well-defined. Let $f=\left(f_{H}\right)_{1<H \leq G}$ be a gluing data. We have to prove that $\psi_{f}:=\psi(f)$ is a morphism of $R \mathcal{D}_{G}$-modules. By the conjugation invariance of the gluing data $f$, it is clear that $\psi_{f}$ commutes with conjugations. To prove that it also commutes with destrictions, let $U / V \preceq M / L$. Then we have

$$
\psi_{f}\left(\operatorname{Des}_{U / V}^{M / L} 1_{M / L}\right)=\psi_{f}\left(1_{U / V}\right)=\operatorname{Res}_{U / V}^{N_{G}(V) / V} f_{V}
$$

We also have

$$
\operatorname{Des}_{U / V}^{M / L} \psi_{f}\left(1_{M / L}\right)=\operatorname{Des}_{U / V}^{M / L} \operatorname{Res}_{M / L}^{N_{G}(L) / L} f_{L}=\operatorname{Des}_{U / V}^{N_{G}(L) / L} f_{L} .
$$

Thus, it is sufficient to show that

$$
\operatorname{Res}_{U / V}^{N_{G}(V) / V} f_{V}=\operatorname{Des}_{U / V}^{N_{G}(L) / L} f_{L}
$$

This follows from the destriction invariance of the gluing data. Indeed, since $L \unlhd V$, the destriction invariance gives

$$
\operatorname{Des}_{N_{G}(V, L) / V}^{N_{G}(L) / L} f_{L}=\operatorname{Res}_{N_{G}(V, L) / V}^{N_{G}(V) / V} f_{V}
$$

Moreover, since $L \unlhd U$, we have $U \leq N_{G}(V) \cap N_{G}(L)=N_{G}(V, L)$. Thus the equality (1) holds, as required.

As a consequence of the above result we obtain the following.

Proposition 3.4. Let $F$ be a destriction functor or a biset functor for $G$. Let $\mathcal{D}_{G}$ denote the orbit category of sections over the collection of all sections in $G$, and let $T$ denote the restriction of the constant functor to the set of all sections $(U, V)$ with $V=1$. Then, as $R$-modules, we have the following isomorphisms

$$
\operatorname{Obs}(F(G)) \cong \operatorname{Ext}_{R \mathcal{D}_{G}}^{1}(T, F) \text { and } \operatorname{Ker}\left(r_{G}^{F}\right) \cong \operatorname{Hom}_{R \mathcal{D}_{G}}(T, F)
$$

Proof. Consider the diagram

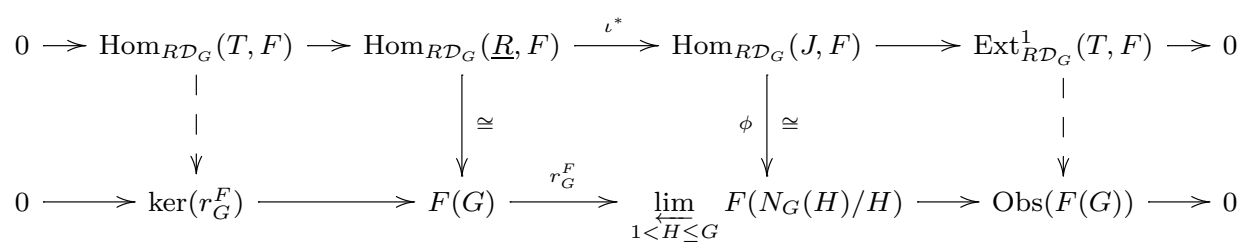

where the vertical maps in the middle are the isomorphisms defined in Lemma 3.1 and Proposition 3.3. The map $\iota^{*}$ is the map induced by the inclusion $\iota: J \rightarrow \underline{R}$. The square in the middle commutes, so it induces two maps, one on each end, which are isomorphisms by 5 -lemma. 
Note that Propositions 3.3 and 3.4 together complete the proof of Theorem 1.3. We now give two examples to illustrate how the obstruction groups $\operatorname{Obs}(F(G))$ can be calculated using specific projective resolutions.

Example 3.5. Let $p$ be a prime, $n \geq 1$, and $G=C_{p^{n}}$ be the cyclic group of order $p^{n}$. Let $Z$ denote the cyclic subgroup of order $p$ in $G$. There is a projective resolution of $T$ as an $R \mathcal{D}_{G}$-module which is of the form

$$
0 \rightarrow P_{(G, Z)} \rightarrow P_{(G, 1)} \rightarrow T \rightarrow 0
$$

Note that in this case $J$ is isomorphic to the projective module $P_{(G, Z)}$. For a biset functor $F$ we have $\operatorname{Ext}_{R \mathcal{D}_{G}}^{i}(T, F)=0$ for $i \geq 2$, and in low dimensions we have a short exact sequence of the form

$$
0 \rightarrow \operatorname{Hom}_{R \mathcal{D}_{G}}(T, F) \rightarrow F(G) \stackrel{\varphi}{\longrightarrow} F(G / Z) \rightarrow \operatorname{Obs}(F(G)) \rightarrow 0
$$

where $\varphi$ is the deflation map $\operatorname{Def}_{G / Z}^{G}$. Since $\operatorname{Def}_{G / Z}^{G} \operatorname{Inf}_{G / Z}^{G}=\operatorname{id}_{F(G / Z)}$, we obtain that in this case $\operatorname{Obs}(F(G))=0$.

The kernel group $\operatorname{ker} r_{G}^{F}$ is not zero in general. If $F=B^{*}$ is the dual group of the Burnside group functor, then we can consider elements $f \in B^{*}(G)$ as functions from the set of subgroups of $G$ to integers, which are constant on conjugacy classes of subgroups. Under this identification, the map $\varphi$ takes $f$ to $f^{\prime}$, where $f^{\prime}(H / Z)=f(H)$ for every $Z \leq H \leq G$. The kernel of $\varphi$ is isomorphic to $\mathbb{Z}$ generated by $e_{1}^{G}$, the function with values $e_{1}^{G}(H)=0$ for $H \neq 1$, and $e_{1}^{G}(1)=1$, which is an idempotent element in $B^{*}(G)$.

Example 3.6. Let $G=D_{8}$ be the dihedral group of order 8. Let $E_{1}, E_{2}$ denote two distinct elementary abelian subgroups of rank 2 , and let $Z$ denote the center of $G$, and let $Q_{1}, Q_{2}$ denote two distinct representatives of the conjugacy classes of the non-central subgroups of order 2. A projective resolution for $T$ as an $R \mathcal{D}_{G}$-module can be given as

$$
0 \rightarrow P_{\left(E_{1}, E_{1}\right)} \oplus P_{\left(E_{2}, E_{2}\right)} \rightarrow P_{\left(E_{1}, Q_{1}\right)} \oplus P_{\left(E_{2}, Q_{2}\right)} \oplus P_{(G, Z)} \rightarrow P_{(G, 1)} \rightarrow T \rightarrow 0
$$

and the cochain complex $\operatorname{Hom}_{R \mathcal{D}_{G}}\left(P_{*}, F\right)$ is of the form

$$
0 \rightarrow F(G) \rightarrow F(G / Z) \oplus F\left(E_{1} / Q_{1}\right) \oplus F\left(E_{2} / Q_{2}\right) \rightarrow F\left(E_{1} / E_{1}\right) \oplus F\left(E_{2} / E_{2}\right) \rightarrow 0
$$

From this we obtain that $\operatorname{Ext}_{R \mathcal{D}_{G}}^{i}(T, F)=0$ for $i \geq 3$. Later we will see that when $G$ is a $p$-group, $\operatorname{Ext}_{R \mathcal{D}_{G}}^{i}(T, F)=0$ for $i \geq \operatorname{rk}(G)+1$ (see Proposition 5.4). Note that the projective resolution that we give above comes from a projective resolution over the Quillen category (see Example 5.5). 


\section{Reduction to smaller collections}

Let $G$ be a finite group and $\mathcal{C}$ be an arbitrary collection of sections of $G$. For an $R \mathcal{D}_{G}$-module $F$, the ext-group $\operatorname{Ext}_{R \mathcal{D}_{G}}^{n}\left(T^{\mathcal{C}}, F\right)$ is defined to be the $n$-th cohomology group of the cochain complex $\operatorname{Hom}_{R \mathcal{D}_{G}}\left(P_{*}, F\right)$ where $P_{*}$ is a projective resolution

$$
\cdots \longrightarrow P_{2} \longrightarrow P_{1} \longrightarrow P_{0} \longrightarrow T^{\mathcal{C}} \longrightarrow 0
$$

of $T^{\mathcal{C}}$ as an $R \mathcal{D}_{G}$-module.

The constant functor $\underline{R} \cong P_{(G, 1)}$ is a projective $R \mathcal{D}_{G^{-m o d u l e}}$, and $T^{\mathcal{C}}=\underline{R} / J^{\mathcal{C}}$ by definition, hence we may assume that the first step of a projective resolution for $T^{\mathcal{C}}$ is the sequence $0 \rightarrow J^{\mathcal{C}} \rightarrow \underline{R} \rightarrow T^{\mathcal{C}} \rightarrow 0$. If we take a projective resolution $Q_{*} \rightarrow J^{\mathcal{C}}$ for $J^{\mathcal{C}}$ as an $R \mathcal{D}_{G}$-module, then by adding the constant functor $\underline{R}$, we obtain a projective resolution for $T^{\mathcal{C}}$ of the form

$$
\cdots \longrightarrow Q_{2} \longrightarrow Q_{1} \longrightarrow Q_{0} \longrightarrow \underline{R} \longrightarrow T^{\mathcal{C}} \longrightarrow 0
$$

From this we can conclude that for every $R \mathcal{D}_{G}$-module $F$,

$$
\operatorname{Ext}_{R \mathcal{D}_{G}}^{n}\left(T^{\mathcal{C}}, F\right) \cong \operatorname{Ext}_{R \mathcal{D}_{G}}^{n-1}\left(J^{\mathcal{C}}, F\right)
$$

for $n \geq 2$. If $\mathcal{C}$ is a collection closed under taking subsections, a projective resolution of $J^{\mathcal{C}}$ as an $R \mathcal{D}_{G}^{\mathcal{C}}$-module is also a projective resolution of $J^{\mathcal{C}}$ as an $R \mathcal{D}_{G}$-module. This means that we can replace the ext-group above with the ext-group $\operatorname{Ext}_{R \mathcal{D}_{G}^{\mathcal{C}}}^{n-1}\left(J^{\mathcal{C}}, F\right)$.

As an $R \mathcal{D}_{G}^{\mathcal{C}}$-module $J^{\mathcal{C}}$ is equal to the constant functor $\underline{R}$ of the category $\mathcal{D}_{G}^{\mathcal{C}}$ (which is also equal to, with abuse of notation, the restriction of the constant functor $\underline{R}$ of the category $\mathcal{D}_{G}$ to the subcategory $\mathcal{D}_{G}^{\mathcal{C}}$ ). Recall that the cohomology of the category $\mathcal{D}_{G}^{\mathcal{C}}$ with coefficients in an $R \mathcal{D}_{G}^{\mathcal{C}}$-module $F$ is defined by

$$
H^{*}\left(\mathcal{D}_{G}^{\mathcal{C}} ; F\right):=\operatorname{Ext}_{R \mathcal{D}_{G}^{\mathcal{C}}}^{*}(\underline{R}, F)
$$

where $\underline{R}$ denotes the constant functor for the category $\mathcal{D}_{G}^{\mathcal{C}}$. From these we conclude the following.

Lemma 4.1. Let $\mathcal{C}$ be a collection of sections of $G$ closed under taking subsections. Then for every $R \mathcal{D}_{G}$-module $F$ we have isomorphisms

$$
\operatorname{Ext}_{R \mathcal{D}_{G}}^{n}\left(T^{\mathcal{C}}, F\right) \cong \operatorname{Ext}_{R \mathcal{D}_{G}}^{n-1}\left(J^{\mathcal{C}}, F\right) \cong H^{n-1}\left(\mathcal{D}_{G}^{\mathcal{C}} ; F\right)
$$

for every $n \geq 2$. 
In low dimensions we have the 4-term exact sequence as before:

$$
0 \longrightarrow \operatorname{Hom}_{R \mathcal{D}_{G}}\left(T^{\mathcal{C}}, F\right) \longrightarrow F(G) \stackrel{r_{G}^{F}}{\longrightarrow} \lim _{(U, \overleftarrow{V}) \in \mathcal{D}_{G}^{\mathcal{C}}} F(U / V) \longrightarrow \operatorname{Ext}_{R \mathcal{D}_{G}}^{1}\left(T^{\mathcal{C}}, F\right) \longrightarrow 0
$$

Note that the inverse limit term is isomorphic to $\operatorname{Hom}_{R \mathcal{D}_{G}}\left(J^{\mathcal{C}} ; F\right) \cong H^{0}\left(\mathcal{D}_{G} ; F\right)$. To extend this short exact sequence to an arbitrary collection $\mathcal{C}$ of subsections, we define the reduced cohomology of $\mathcal{D}_{G}^{\mathcal{C}}$ as follows.

Definition 4.2. Let $\mathcal{C}$ be an arbitrary collection of sections of $G$. For an $R \mathcal{D}_{G}$-module $F$, the reduced cohomology $\widetilde{H}^{n}\left(\mathcal{D}_{G}^{\mathcal{C}} ; F\right)$ of the category $\mathcal{D}_{G}^{\mathcal{C}}$ is defined as the usual cohomology group $H^{n}\left(\mathcal{D}_{G}^{\mathcal{C}} ; F\right)$ for $n \geq 1$ and, at dimensions $n=-1$ and $n=0$, it is defined as the kernel and cokernel of the map $r_{G}^{F}$. We have an exact sequence of the form

$$
0 \longrightarrow \widetilde{H}^{-1}\left(\mathcal{D}_{G}^{\mathcal{C}} ; F\right) \longrightarrow F(G) \stackrel{r_{G}^{F}}{\longrightarrow} \lim _{(U, \overleftarrow{V}) \in \mathcal{D}_{G}^{\mathcal{C}}} F(U / V) \longrightarrow \widetilde{H}^{0}\left(\mathcal{D}_{G}^{\mathcal{C}} ; F\right) \longrightarrow 0
$$

where $r_{G}^{F}$ is the map that takes $f \in F(G / 1)$ to the tuple $\left(\operatorname{Defres}_{U / V}^{G} f\right)_{U / V \in \mathcal{C}}$.

Alternatively, we can define the reduced cohomology as the cohomology groups of a cochain complex. Given a collection of sections $\mathcal{C}$ of a finite group $G$, let $\mathcal{C}^{\prime}$ denote the set of all sections $(M, L)$ such that $(U, V) \in \mathcal{C}$ for some $(U, V) \preceq(M, L)$. Note that when $\mathcal{C}$ is nonempty (which we always assume), $\mathcal{C}^{\prime}$ includes the section $(G, 1)$. Let $J^{\mathcal{C}^{\prime}}$ denote the constant functor for the category $\mathcal{D}_{G}^{\mathcal{C}^{\prime}}$. Then $J^{\mathcal{C}}$ is a submodule of $J^{\mathcal{C}^{\prime}}$ as an $R \mathcal{D}_{G}^{\mathcal{C}^{\prime}}$-module, in particular, the quotient module $J^{\mathcal{C}^{\prime}} / J^{\mathcal{C}}$ is defined.

If $Q_{*} \rightarrow J^{\mathcal{C}}$ is a projective resolution of $J^{\mathcal{C}}$ as an $R \mathcal{D}_{G}^{\mathcal{C}}$-module, then splicing to it the short exact sequence $0 \rightarrow J^{\mathcal{C}} \rightarrow J^{\mathcal{C}^{\prime}} \rightarrow J^{\mathcal{C}^{\prime}} / J^{\mathcal{C}} \rightarrow 0$, we obtain a resolution of $J^{\mathcal{C}^{\prime}} / J^{\mathcal{C}}$ as an $R \mathcal{D}_{G}^{\mathcal{C}^{\prime}}$-module of the form

$$
\cdots \longrightarrow Q_{2} \longrightarrow Q_{1} \longrightarrow Q_{0} \longrightarrow J^{\mathcal{C}^{\prime} \longrightarrow} J^{\mathcal{C}^{\prime}} / J^{\mathcal{C}} \longrightarrow 0
$$

Since $(G, 1)$ is in the collection $\mathcal{C}^{\prime}$, the constant functor $J^{\mathcal{C}^{\prime}}$ is a projective $R \mathcal{D}_{G}^{\mathcal{C}^{\prime}}$-module, hence the above resolution is a projective resolution of $J^{\mathcal{C}^{\prime}} / J^{\mathcal{C}}$ as an $R \mathcal{D}_{G}^{\mathcal{C}^{\prime}}$-module. Applying $\operatorname{Hom}_{R \mathcal{D}_{G}^{\mathcal{C}^{\prime}}}(-, F)$ to this projective resolution, we get a cochain complex

$$
0 \longrightarrow \operatorname{Hom}_{R \mathcal{D}_{G}^{\mathcal{C}^{\prime}}}\left(J^{\mathcal{C}^{\prime}}, F\right) \longrightarrow \operatorname{Hom}_{R \mathcal{D}_{G}^{\mathcal{C}^{\prime}}}\left(Q_{0}, F\right) \longrightarrow \operatorname{Hom}_{R \mathcal{D}_{G}^{\mathcal{C}^{\prime}}}\left(Q_{1}, F\right) \longrightarrow \cdots
$$

where

$$
\operatorname{Hom}_{R \mathcal{D}_{G}^{\mathcal{C}^{\prime}}}\left(J^{\mathcal{C}^{\prime}}, F\right) \cong F(G / 1)
$$


It is now easy to see that the cohomology of this cochain complex is isomorphic to the reduced cohomology of the category $\mathcal{D}_{G}^{\mathcal{C}}$. This shows that the reduced cohomology $\widetilde{H}^{n}\left(\mathcal{D}_{G}^{\mathcal{C}} ; F\right)$ of the category $\mathcal{D}_{G}^{\mathcal{C}}$ can be described as the cohomology of a cochain complex defined as above.

Remark 4.3. If $\mathcal{C}$ is a collection that is closed under taking subsections, then $\mathcal{C}^{\prime}$ is the collection of all sections of $G$. In this case $J^{\mathcal{C}^{\prime}} / J^{\mathcal{C}}=T^{\mathcal{C}}$, hence the reduced cohomology $\widetilde{H}^{n}\left(\mathcal{D}_{G}^{\mathcal{C}} ; F\right)$ is isomorphic to the ext-group $\operatorname{Ext}_{\mathcal{D}_{G}}^{n+1}\left(T^{\mathcal{C}}, F\right)$ for $n \geq-1$. For an arbitrary collection the reduced cohomology is no longer isomorphic to the ext-groups of $T^{\mathcal{C}}$, instead it is isomorphic to the ext-group

$$
\operatorname{Ext}_{\mathcal{D}_{G}^{\mathcal{C}^{\prime}}}^{n+1}\left(J^{\mathcal{C}^{\prime}} / J^{\mathcal{C}}, F\right)
$$

For an arbitrary collection $\mathcal{C}$ we have the following version of our main result in the previous section.

Proposition 4.4. Let $\mathcal{C}$ be an arbitrary collection of sections of $G$, and $F$ be a destriction functor or a biset functor for $G$ over $R$. Then the obstruction group $\operatorname{Obs}^{\mathcal{C}}(F)$ for the gluing problem over $\mathcal{C}$ is isomorphic to the reduced cohomology group $\widetilde{H}^{0}\left(\mathcal{D}_{G}^{\mathcal{C}} ; F\right)$ and the uniqueness is determined by $\widetilde{H}^{-1}\left(\mathcal{D}_{G}^{\mathcal{C}} ; F\right)$.

In the rest of the section we show that under certain conditions the collection $\mathcal{C}$ can be replaced by a smaller collection of sections without changing its reduced cohomology. To do this we use a theorem by Jackowski and Slominska [13] on the cohomology of categories associated to an isotropy presheaf. We first introduce the necessary definitions to state Jackowski and Slominska's theorem.

Let $G$ be a finite group and $W$ be a $G$-poset. Let $S(G)$ denote the $G$-poset of subgroups of $G$ with the $G$-action given by conjugation, and the partial order is induced by the inclusion of subgroups. A $G$-poset map $d: W \rightarrow S(G)$ is called an isotropy presheaf on $W$ if it satisfies $d(w) \leq G_{w}:=\{g \in G \mid g w=w\}$ for every $w \in W$. Given an isotropy presheaf $d$ on $W$, let $W_{d}$ denote the category whose objects are the elements of $W$ and where the morphisms $w_{1} \rightarrow w_{2}$ are given by right cosets $d\left(w_{2}\right) g$ for all $g \in G$ such that $g w_{1} \leq w_{2}$. Note that if we take $W=S(G)$ and $d$ is the identity map, the category $W_{d}$ is the orbit category of $G$, so this definition is quite natural, and the category $W_{d}$ can be thought of a generalization of the orbit category.

Lemma 4.5. Let $\operatorname{Sect}(G, \mathcal{C})$ denote the $G$-poset of all sections in a collection $\mathcal{C}$, where the $G$-action is given by conjugation. Let $d: \operatorname{Sect}(G, \mathcal{C}) \rightarrow S(G)$ be the $G$-map defined by $d((U, V))=U \in S(G)$. Then $d$ defines an isotropy presheaf on $\operatorname{Sect}(G, \mathcal{C})$ and the category $\operatorname{Sect}(G, \mathcal{C})_{d}$ is isomorphic to the category of sections $\mathcal{D}_{G}^{\mathcal{C}}$.

Proof. For a section $(U, V) \in \mathcal{C}$, the isotropy subgroup of the $G$-action on $(U, V)$ is $N_{G}(U, V)=N_{G}(U) \cap N_{G}(V)$ which includes $U$ as a subgroup. Hence $d: \operatorname{Sect}(G, \mathcal{C}) \rightarrow$ 
$S(G)$ defined by $d((U, V))=U$ is an isotropy presheaf. It follows that the category $\operatorname{Sect}(G, \mathcal{C})_{d}$ is isomorphic to the category $\mathcal{D}_{G}^{\mathcal{C}}$.

We now recall a result on isotropy presheaves. Note that for a poset map $f: X \rightarrow Y$ the comma category $y \backslash f$ is defined as the subposet $y \backslash f:=\{x \in X \mid y \leq f(x)\}$.

Proposition 4.6 (Jackowski-Slominska [13]). Let $W^{\prime} \subseteq W$ be a subposet of $W$, and $i$ : $W^{\prime} \rightarrow W$ denote the inclusion map. Suppose that $d^{\prime}$ and $d$ are isotropy presheaves defined on $W^{\prime}$ and $W$ such that $d^{\prime}=d \circ i$. If for every $w \in W$ the topological realization of the poset $w \backslash i=\left\{w^{\prime} \in W^{\prime} \mid w \leq w^{\prime}\right\}$ is $R$-acyclic, then for every $R W_{d}$-module $F$, there is an isomorphism

$$
H^{*}\left(W_{d} ; F\right) \cong H^{*}\left(W_{d^{\prime}}^{\prime} ; F \circ i_{*}\right)
$$

where $i_{*}: W_{d^{\prime}}^{\prime} \rightarrow W_{d}$ is the functor induced by $i$.

Proof. This isomorphism is stated in [13, Section 6] as Equation (6.A). The proof follows from Theorem 3.4 and Proposition 5.4 in [13].

As a consequence of Lemma 4.5 and Proposition 4.6 we obtain the following.

Proposition 4.7. Let $G$ be a finite group, and let $\mathcal{C}^{\prime}$ and $\mathcal{C}$ be two families of sections of $G$ such that $\mathcal{C}^{\prime} \subseteq \mathcal{C}$. Let $i: \operatorname{Sect}\left(G, \mathcal{C}^{\prime}\right) \rightarrow \operatorname{Sect}(G, \mathcal{C})$ denote the inclusion of posets map, and $i_{*}: \mathcal{D}_{G}^{\mathcal{C}^{\prime}} \rightarrow \mathcal{D}_{G}^{\mathcal{C}}$ be the induced map. Suppose that for every $w \in \mathcal{C}$ the poset $w \backslash i=\left\{w^{\prime} \in \mathcal{C}^{\prime} \mid w \leq w^{\prime}\right\}$ has a contractible realization. Then, for every $R \mathcal{D}_{G}^{\mathcal{C}}$-module $F$, there is an isomorphism

$$
\widetilde{H}^{*}\left(\mathcal{D}_{G}^{\mathcal{C}} ; F\right) \cong \widetilde{H}^{*}\left(\mathcal{D}_{G}^{\mathcal{C}^{\prime}} ; F \circ i_{*}\right)
$$

In particular, for a biset functor $F$ for $G$, we have $\operatorname{Obs}^{\mathcal{C}}(F(G)) \cong \operatorname{Obs}^{\mathcal{C}^{\prime}}(F(G))$.

Proof. Let $W=\operatorname{Sect}(G, \mathcal{C})$ and $W^{\prime}=\operatorname{Sect}\left(G, \mathcal{C}^{\prime}\right)$, and let $d$ denote the isotropy presheaf defined on $W$ by $d(U, V)=U$ and let $d^{\prime}=d \circ i$. By Lemma 4.5 and Proposition 4.6, we have an isomorphism $H^{*}\left(\mathcal{D}_{G}^{\mathcal{C}} ; F\right) \cong H^{*}\left(\mathcal{D}_{G}^{\mathcal{C}^{\prime}} ; F \circ i_{d}\right)$. The result for the reduced cohomology follows from this by applying the 5-lemma on the long exact sequence for the cohomology of a pair of categories.

Now we give two applications of Proposition 4.7. Let $G$ be a finite group and $p$ be a fixed prime. Let $\mathcal{D}_{G}^{p}$ denote the category of sections over the collection of sections $(P, Q)$ in $G$ such that $P$ is a $p$-group and $Q \neq 1$, and let $\mathcal{D}_{G}^{e}$ denote the full subcategory of $\mathcal{D}_{G}^{p}$ whose objects are sections of the form $(U, E)$ where $U$ is a $p$-subgroup of $G$ and $E$ is a nontrivial elementary abelian $p$-subgroup of $G$ such that $U \leq C_{G}(E)$. 
Proposition 4.8. For every $R \mathcal{D}_{G}^{p}$-module $F$, there is an isomorphism

$$
\widetilde{H}^{*}\left(\mathcal{D}_{G}^{p} ; F\right) \cong \widetilde{H}^{*}\left(\mathcal{D}_{G}^{e} ; F \circ i_{*}\right)
$$

Proof. Let $W^{p}$ and $W^{e}$ denote the posets corresponding to the collections $\mathcal{C}^{p}$ and $\mathcal{C}^{e}$ for the categories $\mathcal{D}_{G}^{p}$ and $\mathcal{D}_{G}^{e}$, and let $i: W^{e} \rightarrow W^{p}$ denote the inclusion map. The result will follow from Proposition 4.7 once we show that for every $w \in W^{p}$, the poset $w \backslash i$ is contractible. Let $w=(P, Q)$, then $w \backslash i$ is the poset of sections of the form $(U, E)$ such that $1 \neq E \leq Q \leq P \leq U \leq C_{G}(E)$. Since $P \leq C_{G}(E)$, the subgroup $E$ is central in $P$ and it lies in $Q$. Let $Z$ denote the subgroup $\Omega_{1}(Z(P)) \cap Q$, where $\Omega_{1}(Z(P))$ denotes the subgroup of $P$ formed by elements of $Z(P)$ dividing $p$. Note that $Z \neq 1$ because $Q$ is normal in $P$. In particular, $w \backslash i$ is not empty. For every $(U, E)$ in $w \backslash i$, we have

$$
E \leq Z \leq Q \leq P \leq U
$$

hence $(P, Z)$ lies in $w \backslash i$, and $(U, E) \succeq(P, Z)$ for every $(U, E)$ in $w \backslash i$. By [16, 1.5], this means that the poset $w \backslash i$ is canonically contractible to the element $(P, Z)$.

Let $G$ be a finite group and $F$ be a biset functor for $G$ over $R$. Let $\mathcal{D}_{G}^{*}$ denote the orbit category of sections over all sections $(U, V)$ of $G$ with $V \neq 1$. A series of subgroups $1 \leq L_{0} \unlhd L_{1} \unlhd \cdots \unlhd L_{n} \leq G$ is called a subnormal series in $G$ if $L_{i-1} \unlhd L_{i}$ for every $i \in\{1, \ldots, n\}$. The normalizer of a subnormal series $1 \leq L_{0} \unlhd L_{1} \unlhd \cdots \unlhd L_{n} \leq G$ is the intersection of the normalizers $N_{G}\left(L_{i}\right)$ over all $i$. For finite groups we have the following reduction result.

Proposition 4.9. Let $G$ be a finite group, and $\mathcal{D}_{G}^{s}$ denote the full subcategory of $\mathcal{D}_{G}^{*}$ whose objects are sections of the form $(N, L)$ where $N$ is the normalizer of a subnormal series $1<L_{0} \unlhd L_{1} \unlhd \cdots \unlhd L_{n}=L$ in $G$. Then for any $R \mathcal{D}_{G}$-module $F$, there is an isomorphism

$$
\widetilde{H}^{*}\left(\mathcal{D}_{G}^{*} ; F\right) \cong \widetilde{H}^{*}\left(\mathcal{D}_{G}^{s} ; F \circ i_{*}\right)
$$

Proof. Let $W^{*}$ denote the poset of all sections $(U, V)$ of $G$ with $V \neq 1$, and let $W^{s}$ denote the poset of sections of the form $(N, L)$ where $N$ is the normalizer of a subnormal series $1<L_{0} \unlhd L_{1} \unlhd \cdots \unlhd L_{n}=L$. Let $i: W^{s} \rightarrow W^{*}$ denote the inclusion map. By Proposition 4.7, we only need to show that for every $w=(U, V) \in W^{*}$, the subposet $w \backslash i$ is contractible. If $(N, L)$ belongs to $w \backslash i$ then $N$ normalizes a subnormal series $1<$ $L_{0} \unlhd \cdots \unlhd L_{n}=L$ and $L \leq V \leq U \leq N \leq N_{G}(L)$. This gives in particular that $L \unlhd V$. Let $N^{\prime}=N \cap N_{G}(V)$. Then $\left(N^{\prime}, V\right)$ lies in $W^{s}$, because $N^{\prime}$ is the normalizer of the subnormal series $1<L_{0} \unlhd \cdots \unlhd L_{n} \unlhd L_{n+1}=V$. We have a zig-zag of inclusions $(N, L) \succeq\left(N^{\prime}, V\right) \preceq\left(N_{G}(V), V\right)$ in $w \backslash i$, so the poset $w \backslash i$ is contractible, in two steps, to the section $\left(N_{G}(V), V\right)$ in $w \backslash i$. 
In the next section we give a refinement of Proposition 4.8 for $p$-groups, and use it to calculate the obstruction groups for some well-known biset functors defined on $p$-groups. We believe that Proposition 4.9 is also a very useful reduction for gluing problems involving finite groups, but we will not pursue this direction here.

\section{Higher limits over the Quillen category}

Let $G$ be a finite group, and let $\mathcal{D}_{G}^{*}$ denote the category of sections over the collection $\mathcal{C}^{*}$ of sections $(U, V)$ in $G$ such that $V \neq 1$. If $G$ is a $p$-group, then the collection $\mathcal{C}^{p}$ is equal to $\mathcal{C}^{*}$, hence in this case Proposition 4.8 gives an isomorphism

$$
\widetilde{H}^{*}\left(\mathcal{D}_{G}^{*} ; F\right) \cong \widetilde{H}^{*}\left(\mathcal{D}_{G}^{e} ; F \circ i_{*}\right)
$$

for every $R \mathcal{D}_{G}$-module $F$. It turns out that we can refine this isomorphism further to a smaller collection when $G$ is a $p$-group. Let $\mathcal{D}_{G}^{c}$ denote the full subcategory of $\mathcal{D}_{G}$ over the collection

$$
\mathcal{C}^{c}=\left\{\left(C_{G}(E), E\right) \mid E \neq 1 \text { is an elementary abelian } p \text {-subgroup in } G\right\} .
$$

Note that the $\mathcal{D}_{G}^{c}$ is the full subcategory of $\mathcal{D}_{G}^{e}$ formed by the maximal elements in $\mathcal{C}^{e}$. We have the following.

Proposition 5.1. Let $G$ be a finite p-group. Then for any $R \mathcal{D}_{G}^{*}$-module $F$, there is an isomorphism

$$
\widetilde{H}^{*}\left(\mathcal{D}_{G}^{*} ; F\right) \cong \widetilde{H}^{*}\left(\mathcal{D}_{G}^{c} ; F \circ j_{*}\right)
$$

where $j_{*}: \mathcal{D}_{G}^{c} \rightarrow \mathcal{D}_{G}^{*}$ is the map induced by the inclusion.

Proof. It is enough to show that for an $R \mathcal{D}_{G}^{e}$-module $F$, there is an isomorphism

$$
\widetilde{H}^{*}\left(\mathcal{D}_{G}^{e} ; F\right) \cong \widetilde{H}^{*}\left(\mathcal{D}_{G}^{c} ; F \circ k_{*}\right)
$$

where $k_{*}: \mathcal{D}_{G}^{c} \rightarrow \mathcal{D}_{G}^{e}$ is the map induced by inclusion. Let $W^{c}$ and $W^{e}$ denote the posets corresponding to the collections for the categories $\mathcal{C}^{c}$ and $\mathcal{C}^{e}$, and let $k: W^{c} \rightarrow W^{e}$ denote the inclusion map. Let $w=(U, V)$, then $w \backslash k$ is the poset of sections of the form $\left(C_{G}(E), E\right)$ such that $E \leq V \leq U \leq C_{G}(E)$. Note that $V$ is also an elementary abelian p-group and $U \leq C_{G}(V)$. This gives

$$
E \leq V \leq U \leq C_{G}(V) \leq C_{G}(E)
$$

and hence $\left(C_{G}(V), V\right) \preceq\left(C_{G}(E), E\right)$ for every $\left(C_{G}(E), E\right)$ in $w \backslash k$. This means that the poset $w \backslash j$ is canonically contractible to the element $\left(C_{G}(V), V\right)$. Hence the result follows by Proposition 4.7. 
Remark 5.2. Note that when $G$ is not a $p$-group the above argument fails because in that case the pair $\left(C_{G}(V), V\right)$ may not lie in the collection $\mathcal{C}^{p}$ since $C_{G}(V)$ is not necessarily a $p$-group.

Let $G$ be a finite group, and $\mathcal{C}$ be a collection of elementary abelian $p$-subgroups in $G$. The Quillen category $\mathcal{A}_{p}^{\mathcal{C}}(G)$ is defined as the category of elementary abelian subgroups in $\mathcal{C}$ with morphisms $E_{1} \rightarrow E_{2}$ given by compositions of conjugations and inclusions. If $\mathcal{C}$ is the collection of all nontrivial elementary abelian $p$-subgroups in $G$, then we denote the Quillen category by $\mathcal{A}_{p}(G)$, or simply by $\mathcal{A}_{p}$ if $G$ is clear from the context. If we also add the trivial subgroup to the collection of subgroups, we denote the Quillen category by $\mathcal{A}_{p}^{\prime}$.

For an $R \mathcal{D}_{G}$-module $F$, we can define a covariant $R \mathcal{A}_{p}^{\prime}$-module $\bar{F}$ by taking $\bar{F}(E)=$ $F\left(C_{G}(E) / E\right)$ for every $E \in \mathcal{A}_{p}^{\prime}$. For an $\mathcal{A}_{p}^{\prime}$-module $M$, we define the reduced cohomology $\widetilde{H}^{i}\left(\mathcal{A}_{p} ; M\right)$ as the cohomology of the cochain complex obtained by applying $\operatorname{Hom}_{R \mathcal{A}_{p}^{\prime}}(-, M)$ to the resolution

$$
\cdots \rightarrow P_{2} \rightarrow P_{1} \rightarrow P_{0} \rightarrow \underline{R} \rightarrow 0
$$

where $P_{*}$ is the projective resolution for the constant functor $J$ for $\mathcal{A}_{p}$ and $\underline{R}$ is the constant functor for the category $\mathcal{A}_{p}^{\prime}$. Note that $\underline{R}$ is a projective $\mathcal{A}_{p}^{\prime}$-module because it is isomorphic to the projective module $P_{1}$ defined by $P_{1}(-)=R \operatorname{Hom}_{\mathcal{A}_{p}^{\prime}}(1,-)$.

Now we are ready to prove Theorem 1.4 stated in the introduction.

Proposition 5.3. Let $G$ be a finite p-group. Then, the opposite category $\overline{\mathcal{D}}_{G}^{c}$ is isomorphic to the category $\mathcal{A}_{p}$ via the functor which takes $E \in \mathcal{A}_{p}$ to $\left(C_{G}(E), E\right) \in \mathcal{D}_{G}^{c}$. As a consequence, for any $R \mathcal{D}_{G}$-module $F$, there is an isomorphism

$$
\widetilde{H}^{*}\left(\mathcal{D}_{G}^{*} ; F\right) \cong \widetilde{H}^{*}\left(\mathcal{A}_{p} ; \bar{F}\right)
$$

In particular, $\operatorname{Obs}(F(G)) \cong \widetilde{H}^{0}\left(\mathcal{A}_{p} ; \bar{F}\right)$.

Proof. The morphism set $\operatorname{Hom}_{\mathcal{A}_{p}}\left(E_{1}, E_{2}\right)$ can be identified with the set of cosets $g C_{G}\left(E_{1}\right)$ such that ${ }^{g} E_{1} \leq E_{2}$. We can identify this set with the set of morphisms

$$
\operatorname{Hom}_{\mathcal{D}_{G}}\left(\left(C_{G}\left(E_{2}\right), E_{2}\right),\left(C_{G}\left(E_{1}\right), E_{1}\right)\right)=\left\{\left.C_{G}\left(E_{1}\right) x\right|^{x}\left(C_{G}\left(E_{2}\right), E_{2}\right) \preceq\left(C_{G}\left(E_{1}\right), E_{1}\right)\right\}
$$

via the bijection which takes $g C_{G}\left(E_{1}\right)$ to $C_{G}\left(E_{1}\right) g^{-1}$. The second statement follows from Proposition 5.1.

The higher limits over the Quillen category of a finite group $G$ can be calculated using a cochain complex defined by Oliver [15, Theorem 1]. This cochain complex is defined using Steinberg modules and it vanishes at dimensions above the $p$-rank of $G$. Recall that $p$-rank of a finite group $G$, denoted by $\operatorname{rk}_{p}(G)$, is defined as the largest integer $s$ 
such that $(\mathbb{Z} / p)^{s} \leq G$. The rank of a finite group $G$, denoted by $\operatorname{rk}(G)$, is defined as the maximum value of $p$-ranks of $G$ over all primes $p$ dividing the order of $G$. For a $p$-group $G$, the $p$-rank and the rank are equal.

For an elementary abelian $p$-subgroup $E$ of $G$, let $\operatorname{Aut}_{G}(E)$ denote the quotient group $N_{G}(E) / C_{G}(E)$. The Steinberg module $\mathrm{St}_{E}$ is the $\operatorname{Aut}_{G}(E)$-module defined as the reduced homology of the poset of proper subgroups of $E$. As a direct consequence of Theorem 5.3, Oliver's theorem gives the following proposition. A commutative ring $R$ with unity is called $p$-local if for every integer $q$ with $(p, q)=1$, the map $m_{q}: R \rightarrow R$ defined by multiplication by $q$ is an isomorphism.

Proposition 5.4. Let $G$ be a finite p-group, $R$ be a p-local commutative ring, and $F$ be an $R \mathcal{D}_{G}$-module. For each $k \geq 0$, let $\mathcal{E}_{k}$ denote a set of representatives of the conjugacy classes of elementary abelian p-subgroups in $G$ with $\operatorname{rk}(E)=k$ (for $k=0, \mathcal{E}_{0}$ consists of the trivial group). Then for any $i \geq-1$, the reduced cohomology group $\widetilde{H}^{i}\left(\mathcal{D}_{G} ; F\right)$ is isomorphic to the $i$-th cohomology of the cochain complex $\left(C_{\mathrm{St}}^{*}(F), \delta\right)$, where

$$
C_{\mathrm{St}}^{i}(F) \cong \prod_{E \in \mathcal{E}_{i+1}} \operatorname{Hom}_{\mathrm{Aut}_{G}(E)}\left(\mathrm{St}_{E}, F\left(C_{G}(E) / E\right)\right)
$$

In particular, $\widetilde{H}^{i}\left(\mathcal{D}_{G} ; F\right)=0$ for $i \geq \operatorname{rk}(G)$.

The $\operatorname{Aut}_{G}(E)$-action on $F\left(C_{G}(E) / E\right)$ is given by the map induced by conjugation homomorphism $c_{n}: C_{G}(E) / E \rightarrow C_{G}(E) / E$ defined by $c_{n}(x)=n x n^{-1}$ for every $x \in$ $C_{G}(E)$ and $n \in N_{G}(E)$. The boundary maps $\delta$ can be described as follows: For each $E \in \mathcal{A}_{p}$, there is a map

$$
R_{E}: \mathrm{St}_{E} \rightarrow \bigoplus_{\substack{A \leq E \\[E: A]=p}} \mathrm{St}_{A}
$$

defined by truncating chains of subgroups or using the boundary maps for cohomology of posets (see [15, page 1390]). Then the map

$$
\delta^{i-1}: C_{\mathrm{St}}^{i-1}(F) \rightarrow C_{\mathrm{St}}^{i}(F)
$$

is defined as the map that takes $c \in C_{\mathrm{St}}^{i-1}(F)$ to the element whose coordinate for $E \in \mathcal{E}_{i+1}$ is equal to the composite

$$
\mathrm{St}_{E} \stackrel{R_{E}}{\longrightarrow} \bigoplus_{\substack{A \leq E \\[E: \bar{A}]=p}} \operatorname{St}_{A} \stackrel{\oplus c(A)}{\longrightarrow} \bigoplus_{\substack{A \leq E \\[E: \bar{A}]=p}} F\left(C_{G}(A) / A\right) \stackrel{\oplus \text { Defres }}{\longrightarrow} F\left(C_{G}(E) / E\right)
$$

The following example shows how we can calculate the cohomology of $\mathcal{D}_{G}$ using the cochain complex in the theorem. 
Example 5.5. Let $G=D_{8}$ be the dihedral group of order 8 as in Example 3.6. In this case

$$
\operatorname{Hom}_{\mathrm{Aut}_{G}(E)}\left(\mathrm{St}_{E}, F\left(C_{G}(E) / E\right)\right) \cong F\left(C_{G}(E) / E\right)
$$

for every elementary abelian subgroup $E \leq G$. So the cochain complex given in Proposition 5.4 is of the form

$$
0 \rightarrow F(G) \rightarrow F(G / Z) \oplus F\left(E_{1} / Q_{1}\right) \oplus F\left(E_{2} / Q_{2}\right) \rightarrow F\left(E_{1} / E_{1}\right) \oplus F\left(E_{2} / E_{2}\right) \rightarrow 0
$$

This is the same as the cochain complex in Example 3.6 obtained from the projective resolution. The category $\mathcal{A}_{p}^{\prime}$ is also an EI-category so we can write a projective resolution of $\mathcal{A}_{p}^{\prime}$-modules that would give this cochain complex when we apply $\operatorname{Hom}_{R \mathcal{A}_{p}^{\prime}}\left(P_{*}, \bar{F}\right)$ to this resolution. For each $E$, let $P_{E}$ be the projective module where $P_{E}(-)=R \operatorname{Hom}_{\mathcal{A}_{p}^{\prime}}(E,-)$. Let $T$ denote the $R \mathcal{A}_{p}^{\prime}$-module which has value $R$ at the trivial subgroup and zero everywhere else. Then there is a projective resolution for $T$ of the form

$$
0 \rightarrow P_{E_{1}} \oplus P_{E_{2}} \rightarrow P_{Q_{1}} \oplus P_{Q_{2}} \oplus P_{Z} \rightarrow P_{1} \rightarrow T \rightarrow 0
$$

When $R$ is not $p$-local there is a spectral sequence from which one can still calculate the cohomology groups $\widetilde{H}^{*}\left(\mathcal{D}_{G} ; F\right)$ (see [15, Proposition 6]). Higher limits over $\mathcal{A}_{p}(G)$ can also be calculated using a theorem of Grodal [11, Thm 6.1]. As before we assume that $R$ is a $p$-local commutative ring, and $\mathcal{C}$ is a collection of elementary abelian $p$-subgroups closed under taking overgroups. Associated to an $R \mathcal{A}_{p}^{\mathcal{C}}(G)$-module $M$, there is a $G$-local coefficient system $\mathcal{F}$ on $|\mathcal{C}|$ defined by $\mathcal{F}\left(E_{0} \leq \cdots \leq E_{n}\right)=M\left(E_{n}\right)$. Grodal's theorem states that for every $i \geq 0$, there is an isomorphism

$$
H^{i}\left(\mathcal{A}_{p}^{\mathcal{C}}(G) ; M\right) \cong H_{G}^{i}(|\mathcal{C}| ; \mathcal{F})
$$

where the second cohomology group is the equivariant cohomology of the simplicial complex $|\mathcal{C}|$ with the $G$-local coefficient system $\mathcal{F}$. This isomorphism can be extended to reduced cohomology groups by an easy diagram chasing argument. We obtain the following.

Proposition 5.6. Let $G$ be a finite -group, and $R$ be a p-local commutative ring. Let $\mathcal{C}$

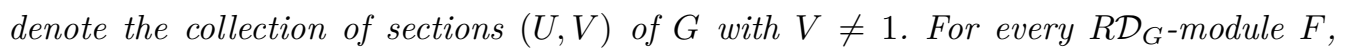
there is an isomorphism

$$
\widetilde{H}^{*}\left(\mathcal{D}_{G}^{\mathcal{C}} ; F\right) \cong \widetilde{H}_{G}^{*}(|\mathcal{C}| ; \mathcal{F})
$$

As in Proposition 5.4 we obtain that the reduced cohomology groups $\widetilde{H}^{i}\left(\mathcal{D}_{G} ; F\right)$ are zero for all $i \geq \operatorname{rk}(G)$. We also obtain the following. 
Proposition 5.7. Let $G$ be a finite p-group and $R$ be a p-local commutative ring. Let $\underline{R}$ denote the constant $R \mathcal{D}_{G}$-module. Then $\widetilde{H}^{n}\left(\mathcal{D}_{G}^{*} ; \underline{R}\right)=0$ for all $n$.

Proof. Let $\mathcal{E}$ denote the collection of all nontrivial elementary abelian $p$-subgroups in $G$. By Proposition 5.6, we have

$$
\widetilde{H}^{*}\left(\mathcal{D}_{G}^{*} ; \underline{R}\right) \cong \widetilde{H}_{G}^{*}(|\mathcal{E}| ; \underline{R}) \cong \widetilde{H}^{*}(|\mathcal{E}| / G ; R) .
$$

The topological realization of $\mathcal{E}$ is equivariantly contractible when $G$ is a $p$-group, hence the orbit space $|\mathcal{E}| / G$ is contractible. Thus $\widetilde{H}^{*}\left(\mathcal{D}_{G}^{*} ; \underline{R}\right)=0$.

\section{Gluing rhetorical $p$-biset functors}

Let $R$ be a commutative ring with unity. A biset functor $F$ over $R$ is called a $p$-biset functor if it is defined over the collection of all $p$-groups. The main aim of this section is the calculation of obstruction groups for a certain type of $p$-biset functors, called rhetorical $p$-biset functors. We give the definition for rhetorical biset functors below in Definition 6.4. Some well-known $p$-biset functors, such as the rational representation ring functor $R_{\mathbb{Q}}$ (and its dual $R_{\mathbb{Q}}^{*}$ ) and the functor $D_{t}$ for the torsion part of Dade group, defined over $p$-groups when $p$ is an odd prime, are rhetorical $p$-biset functors.

Let $F$ be a biset functor for a finite group $G$ over $R$. For a normal $\operatorname{subgroup~} N \unlhd G$, let $e_{N}^{G}$ denote the $(G, G)$-biset $\operatorname{Inf}_{G / N}^{G} \operatorname{Def}_{G / N}^{G}$. The biset $e_{N}^{G}$ is an idempotent of $R B(G, G)$ since the composition $\operatorname{Def}_{G / N}^{G} \operatorname{Inf}_{G / N}^{G}$ is the identity $(G / N, G / N)$-biset. This gives a direct sum decomposition

$$
F(G) \cong e_{N}^{G} F(G) \oplus\left(1-e_{N}^{G}\right) F(G)
$$

From [3, Sections 6.2 and 6.3], for any $M, N \unlhd G$, we have $e_{N}^{G} e_{M}^{G}=e_{N M}^{G}$. For each normal subgroup $N \unlhd G$, we define $f_{N}^{G}$ as the idempotent

$$
f_{N}^{G}=\sum_{N \leq M \unlhd G} \mu_{\unlhd G}(N, M) e_{M}^{G}
$$

where $\mu_{\unlhd G}$ denotes the Möbius function for the poset of normal subgroups of $G$. The elements $f_{N}^{G}$ are orthogonal idempotents of $R B(G, G)$, and $\sum_{N \unlhd G} f_{N}^{G}=\mathrm{id}_{G}$. This gives a decomposition for $F(G)$ in the form

$$
F(G) \cong \bigoplus_{N \unlhd G} f_{N}^{G} F(G) .
$$

Definition 6.1. The summand $f_{1}^{G} F(G)$ corresponding to $N=1$ in this decomposition is called the submodule of faithful elements of $F(G)$, denoted $\partial F(G)$. Note that

$$
\partial F(G)=\bigcap_{1<N \unlhd G} \operatorname{ker} \operatorname{Def}_{G / N}^{G} .
$$


From this definition, we immediately see that $\operatorname{ker} r_{G}^{F} \subseteq \partial F(G)$ where $r_{G}^{F}$ is the detection map in the gluing problem for $F$. We conclude the following.

Lemma 6.2. Let $F$ be a biset functor for a finite group $G$. If $\partial F(G)=0$, then $\operatorname{Hom}_{R \mathcal{D}_{G}}(T, F)=\operatorname{ker} r_{G}^{F}=0$.

There are many examples of biset functors where $\partial F(G)$ is not zero. If $F=B^{*}$ is the biset functor of the dual of the Burnside ring, then by [8, Theorem 3.1] for any $p$-group $G$, we have $\operatorname{ker} r_{G}^{B^{*}}=\operatorname{Hom}_{\mathbb{Z D}_{G}}\left(T, B^{*}\right) \cong \mathbb{Z}$, so $\partial B^{*}(G)$ is not zero. If $F=R_{\mathbb{Q}}^{*}$ is the biset functor of the dual of the rational representation ring, then $\operatorname{Hom}_{\mathbb{Z} \mathcal{D}_{G}}\left(T, R_{\mathbb{Q}}^{*}\right)=0$ if $G$ is a $p$-group of rank at least 2 , and it is equal to $\mathbb{Z}$ otherwise (see [8, Sec. 4] for details).

A special case is where $G=E$ is an elementary abelian $p$-group. In this case we have

$$
\operatorname{Hom}_{R \mathcal{D}_{E}}(T, F)=\operatorname{ker} r_{E}^{F}=\partial F(E)
$$

hence the uniqueness of the solution of the gluing problem is equivalent to the existence of faithful elements in $F(E)$. Moreover, in this case there exists a solution for the gluing problem for any biset functor.

Proposition 6.3 (Bouc-Thevenaz [6]). If $F$ is a biset functor defined on an elementary abelian p-group $E$, then $\operatorname{Obs}(F(E))=0$, i.e., there is a solution for the gluing problem for $F$. The solution is unique if and only if $\partial F(E)=0$.

Proof. The proof is the same as proof of [6, Lemma 2.2] given for the functor $D_{t}$.

An important class of $p$-biset functors are rhetorical $p$-biset functors. They are defined as follows.

Definition 6.4. Let $R_{\mathbb{Q}}(H, K)$ denote the representation ring of $\mathbb{Q} H$ - $\mathbb{Q} K$-bimodules, and

$$
\operatorname{Lin}_{\mathbb{Q}}: R \otimes_{\mathbb{Z}} B(H, K) \rightarrow R \otimes_{\mathbb{Z}} R_{\mathbb{Q}}(H, K)
$$

denote the linearization map which takes an $(H, K)$-biset $X$ to the rational permutation bimodule $\mathbb{Q} X . \mathrm{A} \mathbb{Q} G$-Morita functor [12] or a rhetorical biset functor $[1]$ is a biset functor $F$ such that $F(u)=0$ for every $u \in \operatorname{ker} \operatorname{Lin}_{\mathbb{Q}}$.

For rhetorical biset functors we have the following theorem.

Proposition 6.5 (Hambleton-Taylor-Williams [12]). Let $G$ be a p-group and $F$ be a rhetorical biset functor for $G$ over $R$. Assume $G$ has a normal subgroup $K \cong C_{p} \times C_{p}$. Let $C_{0}, C_{1}, \ldots, C_{p}$ be the distinct cyclic subgroups of $K$ and $Z(G)$ denote the center of $G$. 
(i) If $K$ is central, then the following sequence is split exact

$$
0 \longrightarrow F(G) \stackrel{\alpha}{\longrightarrow} F\left(G / C_{0}\right) \oplus F\left(G / C_{1}\right) \oplus \cdots \oplus F\left(G / C_{p}\right) \stackrel{\beta}{\longrightarrow} \oplus_{p} F(G / K) \longrightarrow 0 .
$$

(ii) If $K$ is not central, we may assume that $K \cap Z(G)=C_{0}$. Let $G_{0}$ denote the centralizer of $K$ in $G$. Then the following sequence is split exact

$$
0 \longrightarrow F(G) \stackrel{\alpha}{\longrightarrow} F\left(G / C_{0}\right) \oplus F\left(G_{0} / C_{1}\right) \stackrel{\beta}{\longrightarrow} F\left(G_{0} / K\right) \longrightarrow 0 .
$$

The maps $\alpha$ and $\beta$ are defined using deflation and restriction maps (see [12, 1.A.16, 5.A.1, and 5.A.3]). In (i), the map $\alpha$ is given by $\prod_{i} \operatorname{Def}_{G / C_{i}}^{G}$, and $\beta$ is defined as follows: $\left.\beta\right|_{F\left(G / C_{i}\right)}=\operatorname{Def}_{G / K}^{G / C_{i}}$ for $1 \leq i \leq p$, and $\left.\beta\right|_{F\left(G / C_{0}\right)}=-\phi$, where $\phi$ is the composite

$$
F\left(G / C_{0}\right) \rightarrow F(G / K) \rightarrow \oplus_{p} F(G / K)
$$

such that the first map is the deflation map and the second map is the diagonal. In (ii), the map $\alpha$ is defined as $\operatorname{Def}_{G / C_{0}}^{G} \times \operatorname{Defres}_{G_{0} / C_{1}}^{G}$, and $\beta: F\left(G / C_{0}\right) \oplus F\left(G_{0} / C_{1}\right) \rightarrow F\left(G_{0} / K\right)$ is the sum of two maps: $F\left(G_{0} / C_{1}\right) \rightarrow F\left(G_{0} / K\right)$ is the deflation map and the map $F\left(G / C_{0}\right) \rightarrow F\left(G_{0} / K\right)$ is the negative of the map Defres $G_{G_{0} / K} / C_{0}$.

Bouc [4, Theorem 3.1] shows that when $F$ is a rhetorical biset functor, the group $F(G)$ can be expressed as a direct sum $\oplus_{S \in \mathcal{G}} \partial F\left(N_{G}(S) / S\right)$ where $\mathcal{G}$ is a family of subgroups, called a genetic basis. A $p$-biset functor $F$ is called a rational p-biset functor if there exists a genetic basis $\mathcal{G}$ giving a direct sum decomposition for $F(G)$ (see [3, Definition 10.1.3]). Hence every rhetorical $p$-biset functor is rational. The converse is not true in general, but when $p$ is odd, the converse also holds for every $p$-biset functor (see [1, Theorem 1.3]).

The most well-known examples of rhetorical biset functors are the rational representation ring $R_{\mathbb{Q}}$ and its dual $R_{\mathbb{Q}}^{*}$. Subfunctors and quotient functors of rhetorical $p$-biset functors are also rhetorical [1, Lemma 3.2]. Using some exact sequences of $p$-biset functors, one can show that the functor of Borel Smith functions $C_{b}$, the functor of unit group of the Burnside ring $B^{\times}$, and the torsion part of the Dade group $D_{t}^{\Omega}$ are also rhetorical $p$-biset functors (see [7], [1, Section 3]). Therefore results on obstruction groups of rhetorical biset functors will apply to these $p$-biset functors. We now prove some general observations for obstruction groups of rhetorical biset functors.

Proposition 6.6. If $F$ is a rhetorical p-biset functor, then $\operatorname{Obs}(F(G))=0=\operatorname{ker} r_{G}^{F}$ for every p-group $G$ with $\operatorname{rk}(Z(G)) \geq 2$.

Proof. Let $G$ be a $p$-group with $\operatorname{rk}(Z(G)) \geq 2$ and $F$ a rhetorical $p$-biset functor. Consider the cochain complex $C_{\mathrm{St}}^{*}(F)$ introduced in Proposition 5.4. The first three terms of the sequence are given as follows:

$$
0 \longrightarrow F(G) \stackrel{\delta^{-1}}{\longrightarrow} \underset{C \in \mathcal{E}_{1}}{\bigoplus} F\left(C_{G}(C) / C\right) \stackrel{\delta^{0}}{\longrightarrow} \underset{E \in \mathcal{E}_{2}}{\bigoplus} \operatorname{Hom}_{\mathrm{Aut}_{G}(E)}\left(\operatorname{St}_{E}, F\left(C_{G}(E) / E\right)\right) \longrightarrow \cdots
$$


where $\operatorname{Aut}_{G}(E)=N_{G}(E) / C_{G}(E)$. We have $\operatorname{ker} r_{G}^{F} \cong \widetilde{H}^{-1}\left(\mathcal{A}_{p} ; \bar{F}\right)=\operatorname{ker} \delta^{-1}$ and

$$
\operatorname{Obs}(F(G))=\widetilde{H}^{0}\left(\mathcal{A}_{p} ; \bar{F}\right)=\operatorname{ker} \delta^{0} / \operatorname{im} \delta^{-1}
$$

This conclusion holds for a $p$-local commutative ring $R$ by Proposition 5.4. To see that it also holds for an arbitrary commutative ring $R$, consider the spectral sequence

$$
E_{1}^{i, j} \cong \bigoplus_{E \in \mathcal{E}_{i+1}} H^{j}\left(\operatorname{Aut}_{G}(E) ; \operatorname{Hom}_{R}\left(\operatorname{St}_{E}, F\left(C_{G}(E) / E\right)\right)\right) \Rightarrow \widetilde{H}^{i+j}\left(\mathcal{A}_{p} ; \bar{F}\right)
$$

Note that the sequence $C_{\mathrm{St}}^{*}(F)$ is the horizontal line $E_{1}^{*, 0}$ on this spectral sequence. The vertical line at $i=-1$ has all zero entries above zero, so the calculation of the reduced 0-th cohomology group $\widetilde{H}^{0}\left(A_{p} ; \bar{F}\right)$ is not affected by the possibly nonzero terms at other places. In fact, when $G$ is a $p$-group we have $N_{G}(C) / C_{G}(C)=1$, hence in this case we also have $E^{0, j}=0$ for all $j>0$. Therefore even for the calculation of the first cohomology group $\widetilde{H}^{1}\left(\mathcal{A}_{p} ; \bar{F}\right)$ we can use the cochain complex $C_{\mathrm{St}}^{*}(F)$ when $G$ is a $p$-group and $R$ is an arbitrary commutative ring.

Let $K$ be a central subgroup isomorphic to $C_{p} \times C_{p}$. The short exact sequence given in part (i) of Proposition 6.5 is a subcomplex of this cochain complex. The quotient complex is of the form

$$
0 \longrightarrow 0 \longrightarrow \bigoplus_{C \in \mathcal{E}_{1}^{\prime}} F\left(C_{G}(C) / C\right) \stackrel{\bar{\delta}^{0}}{\longrightarrow} \bigoplus_{E \in \mathcal{E}_{2}^{\prime}} \operatorname{Hom}_{\operatorname{Aut}_{G}(E)}\left(\operatorname{St}_{E}, F\left(C_{G}(E) / E\right)\right) \longrightarrow \cdots
$$

where $\mathcal{E}_{1}^{\prime}=\mathcal{E}_{1} \backslash\left\{C_{0}, \ldots, C_{p}\right\}$ and $\mathcal{E}_{2}^{\prime}=\mathcal{E}_{2} \backslash\{K\}$. Note that this quotient complex has the same cohomology groups as the cochain complex $C_{\mathrm{St}}^{*}(F)$. From this one sees easily that $\operatorname{ker} r_{G}^{F}=0$ and $\operatorname{Obs}(F(G))=\widetilde{H}^{0}\left(\mathcal{A}_{p} ; \bar{F}\right)=\operatorname{ker} \bar{\delta}^{0}$.

For each $C \in \mathcal{E}_{1}^{\prime}$, the quotient group $C K / C$ is a central subgroup of $C_{G}(C) / C$ and it is isomorphic to $C_{p} \times C_{p}$. Hence for every $C \in \mathcal{E}_{1}^{\prime}$, there is a short exact sequence of the form

$$
0 \longrightarrow F\left(C_{G}(C) / C\right) \stackrel{\alpha}{\longrightarrow} \bigoplus_{i=0}^{p} F\left(C_{G}(C) / C_{i} C\right) \stackrel{\beta}{\longrightarrow} \oplus_{p} F\left(C_{G}(C) / C K\right) \longrightarrow 0
$$

The $i$-th component of $\alpha$ coincides with the summand of $\bar{\delta}^{0}$ corresponding to the index pair $(C, E)$ where $E=C C_{i} \in \mathcal{E}_{2}^{\prime}$. If $C$ and $C^{\prime}$ are two different conjugacy class representatives in $\mathcal{E}_{1}^{\prime}$ such that $C C_{i}=C^{\prime} C_{j}$, then $i=j$, and $C$ and $C^{\prime}$ are two subgroups of $E=C C_{i}$ which are not conjugate to each other. Thus we must have $\operatorname{Aut}_{G}(E)=N_{G}(E) / C_{G}(E)=1$, and under $\bar{\delta}^{0}$ the elements in $F\left(C_{G}(C) / C\right)$ and $F\left(C_{G}\left(C^{\prime}\right) / C^{\prime}\right)$ are mapped to two different summands in

$$
\operatorname{Hom}_{\mathrm{Aut}_{G}(E)}\left(\mathrm{St}_{E}, F\left(C_{G}(E) / E\right)\right) \cong \mathrm{St}_{E}^{*} \otimes F\left(C_{G}(E) / E\right) \cong \oplus_{p} F\left(C_{G}(E) / E\right) .
$$

We conclude that $\operatorname{Obs}(F(G))=\operatorname{ker} \bar{\delta}^{0}=0$. 
Now we consider the case where $G$ is a finite $p$-group and $Z(G)$ is cyclic. In this case $G$ has a normal subgroup isomorphic to $C_{p} \times C_{p}$ or $G$ is a Roquette group, i.e., $G$ is a cyclic group $C_{p^{n}}$, for $n \geq 0$, or generalized quaternion group $Q_{2^{n}}$, for $n \geq 3$, or dihedral group $D_{2^{n}}$, for $n \geq 4$, or a semi-dihedral group $S D_{2^{n}}$, for $n \geq 4$ (see [3, Section 9.3]). If $G$ is a Roquette group, then it is easy to write explicit projective resolutions and calculate $\operatorname{Obs}(F(G))$. If $G$ has a normal subgroup $K \cong C_{p} \times C_{p}$, then $Z=K \cap Z(G)$ is a central subgroup of order $p$. If $A_{1}, A_{2}, \ldots, A_{p}$ denote the subgroups of $K$ which are not central in $G$, then $H=C_{G}(K)=C_{G}\left(A_{i}\right)$ is a subgroup of $G$ of index $p$ for all $i$. Note that if $g \in G \backslash H$, then $g$ permutes the subgroups $\left\{A_{1}, \ldots, A_{p}\right\}$.

Let $\mathcal{A}_{\geq 2}(G)$ denote the poset of elementary abelian subgroups of $G$ of rank greater than or equal to 2 . This poset is non-empty if and only if $\operatorname{rk}(G) \geq 2$. If $\operatorname{rk}(Z(G)) \geq 2$, then $\mathcal{A}_{\geq 2}(G)$ is contractible to a central subgroup $K \cong C_{p} \times C_{p}$ by the canonical contractions $K \leq K E \geq E$. If $\operatorname{rk}(Z(G))=1$, i.e., $Z(G)$ is cyclic, then there is a connected component $\mathcal{B}(G)$ in $\mathcal{A}_{\geq 2}(G)$ such that any connected component of $\mathcal{A}_{\geq 2}(G)$ different from $\mathcal{B}(G)$ consists of a single subgroup of rank 2 (see [10, Lemma 10.21]).

Note that if $\operatorname{rk}(G)=2$, then $A_{\geq 2}(G)$ is just a set of points. In that case, we choose one of the rank 2 elementary abelian subgroups as the component $\mathcal{B}(G)$. If one of these subgroups is normal in $G$, we always choose the normal subgroup as the component $\mathcal{B}(G)$. If $\operatorname{rk}(G) \geq 3$, then $G$ cannot be a Roquette group, hence it has a normal subgroup $E \unlhd G$ of rank 2 . In this case $E$ lies in $\mathcal{B}(G)$. We summarize the properties of the poset $\mathcal{A}_{\geq 2}(G)$ in the following lemma (see [6, Lemma 3.1]).

Lemma 6.7. Let $G$ be a p-group with $\operatorname{rk}(G) \geq 2$ and assume that $Z(G)$ is cyclic.

(a) $G$ has a unique central subgroup $Z$ of order p. Moreover $Z$ is contained in every maximal elementary abelian subgroup of $G$.

(b) There is a connected component $\mathcal{B}(G)$ in $\mathcal{A}_{\geq 2}(G)$ such that any connected component of $\mathcal{A}_{\geq 2}(G)$ different from $\mathcal{B}(G)$ consists of a single subgroup of rank 2 (an isolated vertex). (c) If $E$ is a subgroup in $\mathcal{A}_{\geq 2}(G)-\mathcal{B}(G)$ and if $S$ is a subgroup of $E$ such that $E=Z \times S$, then $N_{G}(S) / S$ is cyclic or generalized quaternion.

(d) If $E$ is a subgroup in $\mathcal{A}_{\geq 2}(G)-\mathcal{B}(G)$, then all the subgroups $S$ of $E$ of order $p$ distinct from $Z$ are conjugate in $G$.

The properties of the poset $\mathcal{A}_{\geq 2}(G)$ can be used to prove the following.

Theorem 6.8. Let $F$ be a rhetorical p-biset functor. Then for every p-group $G$ with $\operatorname{rk}(G) \geq 2$, we have $\operatorname{ker} r_{G}^{F}=0$ and

$$
\operatorname{Obs}(F(G)) \cong \bigoplus_{S \in \mathcal{S}} \partial F\left(C_{G}(S) / S\right)
$$

where the sum is taken over the conjugacy class representatives of cyclic subgroups $S$ such that $E=S \times Z$ belongs to $\mathcal{A}_{\geq 2}(G)-\mathcal{B}(G)$. In particular, if $F$ is such that for every 
cyclic or quaternion group $H, \partial F(H)$ is isomorphic to a fixed abelian group $A$, then

$$
\operatorname{Obs}(F(G)) \cong \widetilde{H}^{0}\left(\mathcal{A}_{\geq 2}(G) / G ; A\right)
$$

Proof. If $\operatorname{rk}(Z(G)) \geq 2$, then $\mathcal{A}_{\geq 2}(G)$ is contractible by a $G$-equivariant homotopy, thus $\mathcal{A}_{\geq 2}(G) / G$ is contractible. Hence in this case the result follows from Lemma 6.6. So we assume that $Z(G)$ is cyclic. The statement is easy to verify for Roquette groups, so let us assume that $G$ has a normal subgroup $K \cong C_{p} \times C_{p}$. Let $Z$ be the central subgroup of $K$ and $A_{1}, \ldots, A_{p}$ denote the non-central subgroups of $K$ of order $p$. Note that $A_{1}, \ldots, A_{p}$ are all conjugate to each other. Let $H=C_{G}(K)$.

Consider the cochain complex $C_{\mathrm{St}}^{*}(F)$. The short exact sequence given in part (ii) of Proposition 6.5, is a subcomplex of this cochain complex. The quotient complex is of the form

$$
0 \longrightarrow 0 \longrightarrow \bigoplus_{C \in \mathcal{E}_{1}^{\prime}} F\left(C_{G}(C) / C\right) \stackrel{\bar{\delta}^{0}}{\longrightarrow} \bigoplus_{E \in \mathcal{E}_{2}^{\prime}} \operatorname{Hom}_{\mathrm{Aut}_{G}(E)}\left(\operatorname{St}_{E}, F\left(C_{G}(E) / E\right)\right) \longrightarrow \cdots
$$

where $\mathcal{E}_{1}^{\prime}=\mathcal{E}_{1} \backslash\left\{Z, A_{1}\right\}$ and $\mathcal{E}_{2}^{\prime}=\mathcal{E}_{2} \backslash\{K\}$. Note that this quotient complex has the same cohomology groups as the cochain complex $C_{\mathrm{St}}^{*}(F)$. From this we obtain that $\operatorname{ker} r_{G}^{F}=0$ and $\operatorname{Obs}(F(G))=\widetilde{H}^{0}\left(\mathcal{A}_{p} ; \bar{F}\right)=\operatorname{ker} \bar{\delta}^{0}$.

Suppose that $C \in \mathcal{E}_{1}^{\prime}$ is such that $E:=C Z$ is not maximal. This means that $E$ belongs to the big component $\mathcal{B}(G)$ of $\mathcal{A}_{\geq 2}(G)$.

Case 1: Assume that $C \leq H=C_{G}(K)$. Then $K$ centralizes $C$, and $K C / C$ is a normal subgroup of $C_{G}(C) / C$ and is isomorphic to $C_{p} \times C_{p}$. If $K C / C$ is central in $C_{G}(C) / C$, then we have a short exact sequence of the form

$$
0 \longrightarrow F\left(C_{G}(C) / C\right) \stackrel{\alpha}{\longrightarrow} F\left(C_{G}(C) / C Z\right) \oplus \underset{i=1}{p} F\left(C_{G}(C) / C A_{i}\right) \stackrel{\beta}{\longrightarrow} \oplus_{p} F\left(C_{G}(C) / C K\right) \longrightarrow 0 .
$$

If $K C / C$ is not central in $C_{G}(C) / C$, then we have a short exact sequence of the form

$$
0 \longrightarrow F\left(C_{G}(C) / C\right) \stackrel{\alpha}{\longrightarrow} F\left(C_{G}(C) / C Z\right) \oplus F\left(C_{G}\left(C A_{1}\right) / C A_{1}\right) \stackrel{\beta}{\longrightarrow} F\left(C_{G}(C K) / C K\right) \longrightarrow 0 .
$$

Note that both of these sequences are subcomplexes of the quotient complex of $C_{\mathrm{St}}^{*}(F)$. So we can take further quotients and assume that the sum in the first term is over the set $\mathcal{E}_{1}^{\prime \prime}$ of conjugacy class representatives of all cyclic subgroups $C$ of $G$ such that $C \not H$.

Case 2: Assume $C$ is such that $C Z$ is not maximal, but $C \not \& H$. Since there is a rank 3 subgroup $E^{\prime} \geq E$, we have $\operatorname{rk}\left(C_{G}(C) / C\right) \geq 2$. Let $\bar{C}_{G}(C):=C_{G}(C) / C$. By induction we assume that $\operatorname{ker} r \bar{C}_{G}(C)=0$. Hence the coboundary map $\delta^{-1}$ for the group $\bar{C}_{G}(C)$ is an injective map

$$
\varphi_{C}: F\left(\bar{C}_{G}(C)\right) \rightarrow \bigoplus_{\bar{D} \in \mathcal{T}_{1}} F\left(C_{\bar{C}_{G}(C)}(\bar{D}) / \bar{D}\right),
$$

where $\mathcal{T}_{1}$ denotes the set of $\bar{C}_{G}(C)$-conjugacy class representatives of subgroups of $\bar{C}_{G}(C)$ such that $\bar{D}=D / C \cong C_{p}$. Since $C$ is not contained in $H$, and $H$ is a subgroup of index $p$ 
in $G$, the subgroup $D$ cannot be a cyclic group of order $p^{2}$, hence we have $D \cong C_{p} \times C_{p}$. Let $B=H \cap D$, then we have $D=B C \cong B \times C$. Note that $N_{G}(D) \leq N_{G}(B)=C_{G}(B)$. This implies that $C_{\bar{C}_{G}(C)}(\bar{D}) / \bar{D} \cong C_{G}(D) / D$. Hence the map

$$
\varphi_{C}: F\left(C_{G}(C) / C\right) \rightarrow \bigoplus_{\bar{D} \in \mathcal{T}_{1}} F\left(C_{G}(D) / D\right)
$$

is injective. Let $\mathcal{E}_{1}^{\prime \prime}$ denote the set of $G$-conjugacy class representatives of cyclic subgroups $C$ of $G$ considered in this case. Then if we take the direct sum of the maps $\varphi_{C}$ over all elements in $\mathcal{E}_{1}^{\prime \prime}$, we get a map from $\oplus_{C \in \mathcal{E}_{1}^{\prime \prime}} F\left(C_{G}(C) / C\right)$ to the group

$$
\bigoplus_{C \in \mathcal{E}_{1}^{\prime \prime}} \bigoplus_{\bar{D} \in \mathcal{T}_{1}} F\left(C_{G}(D) / D\right)=\bigoplus_{D \in \mathcal{E}_{2}^{\prime \prime}} \operatorname{Hom}_{\operatorname{Aut}_{G}(D)}\left(\operatorname{St}_{D}, F\left(C_{G}(D) / D\right)\right)
$$

where $\mathcal{E}_{2}^{\prime \prime}$ is the set of $G$-conjugacy classes of rank 2 elementary abelian subgroups of $G$ such that $D=(D \cap H) C$ with $C Z$ not maximal and $D \not \leq H$. Note that this map appears in the quotient complex of $C_{\mathrm{St}}^{*}(F)$. Hence by an argument similar to the argument above, we can delete summands of the form $F\left(C_{G}(C) / C\right)$ where $E=C Z$ belongs to the big component, but $C \not \leq H$.

The remaining cyclic subgroups $C$ satisfy the conditions (c) and (d) of Lemma 6.7. In particular, $E=C Z$ is a maximal rank 2 subgroup and in $E$ all subgroups of order $p$ distinct from $Z$ are conjugate. For each such $C$, we have a map

$$
F\left(C_{G}(C) / C\right) \rightarrow F\left(C_{G}(C) / C Z\right) .
$$

By condition (c), the quotient group $L=C_{G}(C) / C$ is cyclic or isomorphic to a generalized quaternion group, and the kernel of this map is $\partial F(L)$. Hence we obtain the isomorphism for $\operatorname{Obs}(F(G))$ given in the statement of the theorem. If $\partial F(L)$ is equal to a fixed abelian group $A$, then we obtain

$$
\operatorname{Obs}(F(G)) \cong \widetilde{H}^{0}\left(\mathcal{A}_{\geq 2}(G) / G ; A\right)
$$

In the next section we discuss how this result applies to some $p$-biset functors related to endo-permutation modules and to the dual of the rational representation ring functor.

\section{Gluing endo-permutation modules}

Let $G$ be a $p$-group and $k$ a field of characteristic $p$. A $k G$-module $M$ is called an endo-permutation module if $\operatorname{Hom}_{k}(M, M) \cong M \otimes M^{*}$ is a permutation $k G$-module. An endo-permutation module is capped if $M \otimes M^{*}$ has the trivial module $k$ as a summand. Under a suitably defined equivalence relation on endo-permutation modules, the set of equivalence classes $[M]$ of capped endo-permutation modules forms an abelian group 
called the Dade group of $G$, denoted $D(G)$. The addition in $D(G)$ is defined by $\left[M_{1}\right]+$ $\left[M_{2}\right]=\left[M_{1} \otimes M_{2}\right]$ (see [3, Definition 12.2.8], [9]).

Given a nonempty $G$-set $X$, the kernel of the augmentation map $k X \rightarrow k$ is a capped endo-permutation module if $\left|X^{G}\right| \neq 1$. In this case, the equivalence class of this endopermutation module is called a relative syzygy and is denoted $\Omega_{X}$. The subgroup of $D(G)$ generated by relative syzygies $\Omega_{X}$ over all finite nonempty $G$-sets $X$ with $X^{G}=\emptyset$ is denoted $D^{\Omega}(G)$ (see [3, Definition 12.6.3]). The assignment $G \rightarrow D(G)$ together with suitably defined restriction, induction, isogation, deflation, and inflation maps is not a biset functor because of some Galois twists that occur, however the Dade group of relative syzygies $D^{\Omega}(G)$ is a $p$-biset functor (see [3, Theorem 12.6.8]). It is also known that when $p$ is odd we have $D^{\Omega}=D$, and hence in this case $D$ is also a $p$-biset functor (see [3, Theorem 12.9.10]).

The torsion part of the Dade group $D^{\Omega}$ is a subfunctor of $D^{\Omega}$, denoted $D_{t}^{\Omega}$. Let $B^{*}$ denote the dual of the Burnside ring functor, $R_{\mathbb{Q}}^{*}$ the dual of the rational representation ring functor, and $C_{b}$ the functor for the group of Borel-Smith functions. Then by Theorem 1.2 and 1.3 in [7], there is a commutative diagram of $p$-biset functors of the form

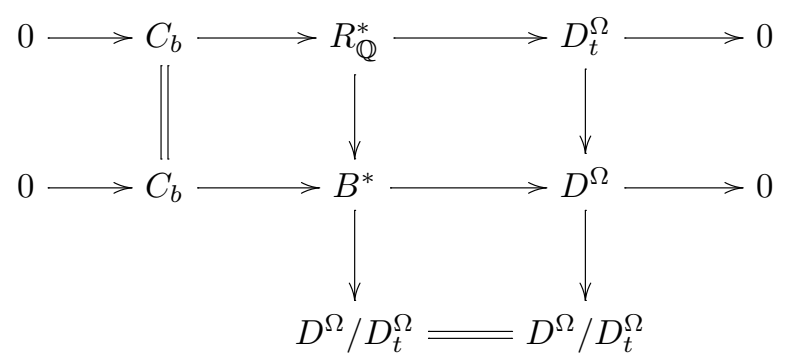

Note that if $p$ is odd, we have $D^{\Omega}=D$.

From this diagram it is clear that the functors $C_{b}$ and $D_{t}^{\Omega}$ are both rhetorical $p$-biset functors since they are subfunctors and quotient functors of the dual of the rational representation ring functor $R_{\mathbb{Q}}^{*}$, which is rhetorical (see [1, Lemma 3.2]). Hence if $F$ is equal to $D_{t}^{\Omega}, C_{b}$, or $R_{\mathbb{Q}}^{*}$, then $\operatorname{Obs}(F(G))=0$ for every $p$-group $G$ with $\operatorname{rk}(Z(G)) \geq 2$. Coşkun uses this calculation to give another proof for the rank calculation for the group of endo-trivial modules $T(G)$ (see [8, Theorem 6.1]).

Now let us assume that $p$ is odd. Then the torsion part of the Dade group $D_{t}$ is equal to $D_{t}^{\Omega}$, hence it is a rhetorical $p$-biset functor. It is also known that $\partial D_{t}(G) \cong \mathbb{Z} / 2$ for every nontrivial cyclic group $G$. So as a corollary of Theorem 6.8, we recover the following theorem of Bouc and Thévenaz [6, Theorem 1.1].

Proposition 7.1 (Bouc-Thévenaz). If $p$ is an odd prime number and $G$ is a p-group that is not cyclic, then there is a short exact sequence of abelian groups 


$$
0 \longrightarrow D_{t}(G) \stackrel{r_{G}^{D_{t}}}{\longrightarrow} \lim _{1<\overleftarrow{K} \leq G} D_{t}\left(N_{G}(H) / H\right) \longrightarrow \widetilde{H}^{0}\left(\mathcal{A}_{\geq 2}(G) ; \mathbb{F}_{2}\right)^{G} \longrightarrow 0
$$

where $H^{0}\left(\mathcal{A}_{\geq 2}(G) ; \mathbb{F}_{2}\right)^{G}$ denotes the group consists of all $G$-invariant cohomology classes under the $G$-action induced by the conjugation action on $\mathcal{A}_{\geq 2}(G)$.

Proof. By Theorem 6.8, the map $r_{G}^{D_{t}}$ is injective and the obstruction group is isomorphic to the reduced cohomology group $\widetilde{H}^{0}\left(\mathcal{A}_{\geq 2}(G) / G ; \mathbb{F}_{2}\right)$. Note that

$$
H^{0}\left(\mathcal{A}_{\geq 2}(G) / G ; \mathbb{F}_{2}\right) \cong H^{0}\left(\mathcal{A}_{\geq 2}(G) ; \mathbb{F}_{2}\right)^{G}
$$

by the definition of the 0-th cohomology. When $p$ is odd, there is always a normal subgroup $K \cong C_{p} \times C_{p}$, therefore there is a $G$-invariant component in $\mathcal{A}_{\geq 2}(G)$. Hence the isomorphism above induces an isomorphism of reduced cohomology classes

$$
\widetilde{H}^{0}\left(\mathcal{A}_{\geq 2}(G) / G ; \mathbb{F}_{2}\right) \cong \widetilde{H}^{0}\left(\mathcal{A}_{\geq 2}(G) ; \mathbb{F}_{2}\right)^{G} \text {. }
$$

For the dual of the rational representation $\operatorname{ring} R_{\mathbb{Q}}^{*}$ we have a similar calculation. For this calculation we do not assume that $p$ is odd. If $H$ is a nontrivial cyclic group or a quaternion group, then $\partial R_{\mathbb{Q}}^{*}(H) \cong \mathbb{Z}$. Hence as a direct consequence of Theorem 6.8, we obtain the following.

Proposition 7.2 (Coşkun [8]). If $G$ is p-group with $\operatorname{rk}(G) \geq 2$, then

$$
\operatorname{Obs}\left(R_{\mathbb{Q}}^{*}(G)\right) \cong \widetilde{H}^{0}\left(\mathcal{A}_{\geq 2}(G) / G ; \mathbb{Z}\right)
$$

This is slightly different than the isomorphism given in [8, Theorem 4.4]. There the obstruction group $\operatorname{Obs}\left(R_{\mathbb{Q}}^{*}\right)$ is given as isomorphic to $\widetilde{H}^{0}\left(\mathcal{A}_{\geq 2}(G) ; p \mathbb{Z}\right)^{G}$ which is isomorphic to $\widetilde{H}^{0}\left(\mathcal{A}_{\geq 2}(G) / G ; \mathbb{Z}\right)$ in all cases except when $G$ is a rank 2 Roquette group, i.e., when $G$ is dihedral or semi-dihedral group. In this case we believe the isomorphism in Proposition 7.2 gives the right answer. Note that since the rational representation ring functor $R_{\mathbb{Q}}$ also has the property $\partial R_{\mathbb{Q}}(H) \cong \mathbb{Z}$ for all $H$ that is cyclic or isomorphic to a generalized quaternion group, we also have

$$
\operatorname{Obs}\left(R_{\mathbb{Q}}(G)\right) \cong \widetilde{H}^{0}\left(\mathcal{A}_{\geq 2}(G) / G ; \mathbb{Z}\right)
$$

for every $p$-group $G$ with $\operatorname{rk}(G) \geq 2$.

Using Theorem 6.8 we can also calculate the obstruction group $\operatorname{Obs}\left(D_{t}^{\Omega}(G)\right)$ when $G$ is a 2-group.

Proposition 7.3. Let $G$ be a 2-group with $\operatorname{rk}(G) \geq 2$. Let $\mathcal{S}$ denote the set of conjugacy class representatives of cyclic subgroups $S$ such that $S \times Z$ belongs to $\mathcal{A}_{\geq 2}(G)-\mathcal{B}(G)$. Then $\operatorname{ker} r_{G}^{D_{t}}=0$ and 


$$
\operatorname{Obs}\left(D_{t}^{\Omega}(G)\right) \cong \bigoplus_{S \in \mathcal{S}} \mathbb{Z} / n_{S} \mathbb{Z}
$$

where

$$
n_{S}= \begin{cases}1 & \text { if } C_{G}(S) / S \cong C_{2} \\ 2 & \text { if } C_{G}(S) / S \cong C_{2^{n}}, n \geq 2 \\ 4 & \text { if } C_{G}(S) / S \cong Q_{2^{n}}, n \geq 3\end{cases}
$$

Proof. This follows from Theorem 6.8 and from the computations of the Dade groups of abelian groups and generalized quaternion groups (see [3, Corollary 12.10.3] and [9, Proposition 12.2]).

Note that the calculation given above is consistent with the earlier calculations done by Coşkun [8, Theorem 5.1] for the Borel-Smith functor $C_{b}$. To see the connection between these two calculations, consider the long exact cohomology sequence for the coefficient sequence $0 \rightarrow C_{b} \rightarrow R_{\mathbb{Q}}^{*} \rightarrow D_{t}^{\Omega} \rightarrow 0$ given as follows:

$$
\cdots \rightarrow \widetilde{H}^{-1}\left(\mathcal{D}_{G}^{*} ; D_{t}^{\Omega}\right) \rightarrow \operatorname{Obs}\left(C_{b}(G)\right) \rightarrow \operatorname{Obs}\left(R_{\mathbb{Q}}^{*}(G)\right) \rightarrow \operatorname{Obs}\left(D_{t}^{\Omega}(G)\right) \rightarrow H^{1}\left(\mathcal{D}_{G}^{*} ; C_{b}\right) \rightarrow \cdots
$$

By Proposition 7.3 we have $\widetilde{H}^{-1}\left(\mathcal{D}_{G}^{*} ; D_{t}^{\Omega}\right)=\operatorname{ker} r_{G}^{D_{t}^{\Omega}}=0$, and

$$
\operatorname{Obs}\left(D_{t}^{\Omega}(G)\right) \cong \bigoplus_{S \in \mathcal{S}} \mathbb{Z} / n_{S} \mathbb{Z}
$$

where $n_{S}$ is defined as above. By Proposition 7.2, we have

$$
\operatorname{Obs}\left(R_{\mathbb{Q}}^{*}(G)\right) \cong \widetilde{H}^{0}\left(\mathcal{A}_{\geq 2}(G) / G ; \mathbb{Z}\right) \cong \bigoplus_{S \in \mathcal{S}} \mathbb{Z}
$$

Hence we recover the obstruction group calculation $\operatorname{Obs}\left(C_{b}(G)\right)=\widetilde{H}_{b}^{0}\left(\mathcal{A}_{\geq 2}(G), p \mathbb{Z}\right)^{G}$ given in [8, Theorem 5.1].

Remark 7.4. The above discussion on the obstruction group for $C_{b}(G)$ gives, in particular, that the map $\operatorname{Obs}\left(R_{\mathbb{Q}}^{*}(G)\right) \rightarrow \operatorname{Obs}\left(D_{t}^{\Omega}(G)\right)$, induced by the map $R_{\mathbb{Q}}^{*} \rightarrow D_{t}^{\Omega}$, is surjective.

The rest of the section is devoted to the calculation of the obstruction group for the Dade group functor $D$ when $p$ is an odd prime. In this case $D=D^{\Omega}$, so we may use the short exact sequence $0 \rightarrow C_{b} \rightarrow B^{*} \rightarrow D^{\Omega} \rightarrow 0$ to calculate the obstruction group. The associated long exact sequence gives a sequence of the following form

$$
\cdots \rightarrow \operatorname{Obs}\left(B^{*}(G)\right) \rightarrow \operatorname{Obs}(D(G)) \stackrel{\delta}{\longrightarrow} H^{1}\left(\mathcal{D}_{G}^{*} ; C_{b}\right) \rightarrow H^{1}\left(\mathcal{D}_{G}^{*} ; B^{*}\right) \rightarrow \cdots
$$

We claim that the connecting homomorphism $\delta$ is an isomorphism. To prove this we first need a lemma. 
Let $\nabla$ denote the destriction algebra and let $\mathrm{c}_{1}^{+}$be the sum of the idempotents $\mathrm{c}_{H / H}$ in $\nabla$ as $H$ runs over all subgroups of $G$. Let $\Pi=\mathrm{c}_{1}^{+} \nabla \mathrm{c}_{1}^{+}$be the truncation of $\nabla$ by $\mathrm{c}_{1}^{+}$. The algebra $\Pi$ can be identified with the subalgebra of $\nabla$ generated by conjugations $\mathrm{c}_{g(H / H), H / H}^{g}$ for all $g \in G$ and $H \leq G$. Moreover, it is easy to see that the algebra $\Pi$ is Morita equivalent to the product $\prod_{H \leq_{G} G} \mathbb{Z}\left[N_{G}(H) / H\right]$ of group algebras.

Lemma 7.5. Let $B^{*}$ denote the p-biset functor of super class functions and let $Z$ be the $\Pi$-module $\prod_{H \leq G} \mathbb{Z}$ with the trivial $\Pi$-action. Then

(1) $B^{*} \cong \operatorname{Coind}_{\Pi}^{\nabla} Z$ as $\nabla$-modules.

(2) $\operatorname{Ext}_{\nabla}^{1}\left(J, B^{*}\right)=0$.

Proof. By definition,

$$
\operatorname{Coind}_{\Pi}^{\nabla} Z=\operatorname{Hom}_{\Pi}(\nabla, Z)=\operatorname{Hom}_{\Pi}\left(\mathrm{c}_{1}^{+} \nabla, Z\right)
$$

where we regard $\nabla$ as a $(\Pi, \nabla)$-bimodule via left and right multiplication. Thus given a subgroup $K \leq G$, we have

$$
\left(\operatorname{Coind}_{\Pi}^{\nabla} Z\right)(K)=\operatorname{Hom}_{\Pi}\left(\mathrm{c}_{1}^{+} \nabla \mathrm{c}_{K}, Z\right)
$$

Now clearly, for any subgroup $H \leq K$, there is a unique element in $\mathrm{c}_{1}^{+} \nabla \mathrm{c}_{K}$ of the form $\operatorname{Des}_{H / H}^{K}$. Hence any $f \in \operatorname{Hom}_{\Pi}\left(\mathrm{c}_{1}^{+} \nabla \mathrm{c}_{K}, Z\right)$ is uniquely determined by its evaluations $f\left(\operatorname{Des}_{H / H}^{K}\right)$ as $H$ runs over conjugacy classes of subgroups of $K$. In particular, we obtain a group homomorphism

$$
\operatorname{Coind}_{\Pi}^{\nabla} Z(K) \rightarrow B^{*}(K), \quad f \mapsto(f(H / H))_{H \leq K}
$$

for any subgroup $K$ of $G$. It is straightforward to check that this is a functorial isomorphism. Hence the first part holds.

To prove the second part, note that since there is the inclusion of rings $\Pi \subseteq \nabla$, we have the base change spectral sequence for ext-groups (see [18, Exercise 5.6.3])

$$
E_{2}^{p, q}=\operatorname{Ext}_{\nabla}^{p}\left(J, \operatorname{Ext}_{\Pi}^{q}(\nabla, Z)\right) \Rightarrow \operatorname{Ext}_{\Pi}^{p+q}(J, Z) .
$$

The exact sequence in low degrees becomes

$$
\begin{gathered}
0 \longrightarrow \operatorname{Ext}_{\nabla}^{1}\left(J, \operatorname{Hom}_{\Pi}(\nabla, Z)\right) \longrightarrow \operatorname{Ext}_{\Pi}^{1}(J, Z) \longrightarrow \operatorname{Hom}_{\nabla}\left(J, \operatorname{Ext}_{\Pi}^{1}(\nabla, Z)\right) \longrightarrow \\
\longrightarrow \operatorname{Ext}_{\nabla}^{2}\left(J, \operatorname{Hom}_{\Pi}(\nabla, Z)\right) \longrightarrow \operatorname{Ext}_{\Pi}^{2}(J, Z) .
\end{gathered}
$$

By the first part, $B^{*}=\operatorname{Hom}_{\Pi}(\nabla, Z)$, hence it is sufficient to show that $\operatorname{Ext}_{\Pi}^{1}(J, Z)$ vanishes. But we have 


$$
\operatorname{Ext}_{\Pi}^{1}(J, Z)=\operatorname{Ext}_{\Pi}^{1}\left(\mathrm{c}_{1}^{+} J, Z\right)=\bigoplus_{1<H \leq{ }_{G} G} \operatorname{Ext}_{\Pi}^{1}\left(\mathrm{c}_{H / H}^{+} J, c_{H / H}^{+} Z\right)
$$

where $\mathrm{c}_{H / H}^{+}=\sum_{K=_{G} H} \mathrm{c}_{K / K}$. Finally, using the above Morita equivalence and the Morita invariance of the ext-groups, we get for any subgroup $H$ of $G$ that

$$
\operatorname{Ext}_{\Pi}^{1}\left(\mathrm{c}_{H / H}^{+} J, \mathrm{c}_{H / H}^{+} Z\right) \cong \operatorname{Ext}_{R N_{G}(H) / H}^{1}(\mathbb{Z}, \mathbb{Z})=H^{1}\left(N_{G}(H) / H ; \mathbb{Z}\right)
$$

which is zero since $N_{G}(H) / H$ is a finite group. Thus $\operatorname{Ext}_{\nabla}^{1}\left(J, B^{*}\right)=0$, as required.

Using this lemma we prove the following.

Proposition 7.6. Let $D$ denote the functor for the Dade group, and $C_{b}$ denote the functor for the group of Borel-Smith functions. Then

$$
\operatorname{Obs}(D(G)) \cong H^{1}\left(\mathcal{D}_{G}^{*} ; C_{b}\right)
$$

Proof. By [8, Theorem 3.1], we have $\operatorname{Obs}\left(B^{*}(G)\right)=0$. By the long exact sequence given in Equation (5), it is enough to show that $H^{1}\left(\mathcal{D}_{G}^{*} ; B^{*}\right)=0$. By Lemma 4.1 , we have

$$
H^{1}\left(\mathcal{D}_{G}^{*} ; B^{*}\right) \cong \operatorname{Ext}_{R \mathcal{D}_{G}}^{1}\left(J, B^{*}\right)
$$

hence the result follows from the Lemma 7.5.

In Bouc [2, Theorem 2.15], it is shown that the obstruction group $\operatorname{Obs}(D(G))$ for a $p$-group $G$, when $p$ is odd, embeds into the group $H^{1}\left(\mathcal{A}_{\geq 2}(G) ; \mathbb{Z}\right)^{(G)}$ which is defined as the quotient group $Z^{1} / B^{1}$ where $Z^{1}$ is the group of $G$-invariant 1-cocycles in $\mathcal{A}_{\geq 2}(G)$ and $B^{1}$ is the subgroup generated by the boundaries of $G$-invariant 0 -chains in $\mathcal{A}_{\geq 2}(G)$. We note the following observation about this group.

Lemma 7.7. The group $H^{1}\left(\mathcal{A}_{\geq 2}(G) ; \mathbb{Z}\right)^{(G)}$ defined in [2, Notation 2.9] is isomorphic to the cohomology group $H^{1}\left(\mathcal{A}_{\geq 2}(G) / G ; \mathbb{Z}\right)$ where $\mathcal{A}_{\geq 2}(G) / G$ denotes the orbit space under the conjugation action.

Proof. Let $X=\mathcal{A}_{\geq 2}(G)$. Note that the group $H^{1}\left(\mathcal{A}_{\geq 2}(G) ; \mathbb{Z}\right)^{(G)}$ defined above is the cohomology group at dimension 1 of the cochain complex

$$
0 \rightarrow \operatorname{Hom}_{\mathbb{Z} G}\left(C_{0}(X), \mathbb{Z}\right) \stackrel{\delta^{0}}{\longrightarrow} \operatorname{Hom}_{\mathbb{Z} G}\left(C_{1}(X), \mathbb{Z}\right) \stackrel{\delta^{1}}{\longrightarrow} \operatorname{Hom}_{\mathbb{Z} G}\left(C_{2}(X), \mathbb{Z}\right) \rightarrow \cdots
$$

Since

$$
\operatorname{Hom}_{\mathbb{Z} G}\left(C_{*}(X), \mathbb{Z}\right) \cong \operatorname{Hom}_{\mathbb{Z}}\left(C_{*}(X)_{G}, \mathbb{Z}\right) \cong \operatorname{Hom}_{\mathbb{Z}}\left(C_{*}(X / G), \mathbb{Z}\right),
$$

the group $H^{1}\left(\mathcal{A}_{\geq 2}(G) ; \mathbb{Z}\right)^{(G)}$ is isomorphic to the cohomology group $H^{1}(X / G ; \mathbb{Z})$. 
In general $\operatorname{Obs}(D(G))$ is not isomorphic to $H^{1}\left(\mathcal{A}_{\geq 2}(G) / G ; \mathbb{Z}\right)$ (see [2, Section 6]). In Theorem 1.5 we give an explicit description of the obstruction group $\operatorname{Obs}(D(G))$ as a subgroup of $H^{1}\left(\mathcal{A}_{\geq 2}(G) / G ; \mathbb{Z}\right)$. Before giving the proof of Theorem 1.5 , we prove a useful technical result.

Proposition 7.8. Let $F$ be a rhetorical p-biset functor such that $\partial F(H)$ is equal to a fixed abelian group A for every group $H$ isomorphic to a nontrivial cyclic group or a generalized quaternion group. Then there is an isomorphism

$$
H^{1}\left(\mathcal{D}_{G}^{*} ; F\right) \cong H^{1}\left(\mathcal{A}_{\geq 2}(G) / G ; A\right) .
$$

Proof. First, let us assume that $\operatorname{rk}(Z(G)) \geq 2$. Let $K \cong C_{p} \times C_{p}$ be a central subgroup in $G$. Then the argument in the proof of Proposition 6.6 gives us that the complex $C_{\mathrm{St}}^{*}(F)$ can be reduced to a complex of the form

$$
0 \stackrel{\bar{\delta}^{0}}{\rightarrow} \bigoplus_{E \in \mathcal{E}_{2}^{\prime \prime}} \operatorname{Hom}_{\operatorname{Aut}_{G}(E)}\left(\operatorname{St}_{E}, F\left(C_{G}(E) / E\right)\right) \stackrel{\bar{\delta}^{1}}{\rightarrow} \bigoplus_{E \in \mathcal{E}_{3}^{\prime \prime}} \operatorname{Hom}_{\operatorname{Aut}_{G}(E)}\left(\operatorname{St}_{E}, F\left(C_{G}(E) / E\right)\right) \longrightarrow \cdots
$$

where $\mathcal{E}_{2}^{\prime \prime}$ is the $G$-conjugacy classes of rank 2 elementary abelian subgroups $E$ such that $E \cap K=1$, and $\mathcal{E}_{3}^{\prime \prime}$ is the $G$-conjugacy classes of rank 3 elementary abelian subgroups $E$ such that $K \not \leq E$. For each $E \in \mathcal{E}_{2}^{\prime \prime}$, we have a short exact sequence of the form

$$
0 \longrightarrow F\left(C_{G}(E) / E\right) \stackrel{\alpha}{\longrightarrow} \bigoplus_{i=0}^{p} F\left(C_{G}(E) / C_{i} E\right) \stackrel{\beta}{\longrightarrow} \oplus_{p} F\left(C_{G}(E) / E K\right) \longrightarrow 0
$$

Note that for each $E$, the rank 3 elementary abelian subgroup $C_{i} E$ does not include $K$. This gives that the map $\bar{\delta}^{1}$ is injective, hence $H^{1}\left(\mathcal{D}_{G}^{*} ; F\right)=0$. Since $K$ is central, $\mathcal{A}_{\geq 2}(G)$ is canonically contractible by equivariant homotopies $K \leq K E \geq E$. Therefore $H^{1}\left(\mathcal{A}_{\geq 2}(G) / G ; A\right)=0$, hence the isomorphism holds in this case.

Now we assume that $Z(G)$ is cyclic. We may also assume that $G$ has a normal subgroup $K \cong C_{p} \times C_{p}$ because otherwise $G$ is a Roquette group and by direct computation we can see that for Roquette groups both cohomology groups are trivial. As before let $Z$ denote the central subgroup of $K$ and $A_{1}, \ldots, A_{p}$ denote the non-central subgroups of $K$ of order $p$. By the argument we used in the proof of Proposition 6.8 we can reduce the complex $C_{\mathrm{St}}^{*}(F)$ to a complex of the form

$$
\begin{aligned}
0 \stackrel{\bar{\delta}^{-1}}{\longrightarrow} \bigoplus_{C \in \mathcal{E}_{1}^{\prime \prime}} F\left(C_{G}(C) / C\right) \stackrel{\bar{\delta}^{0}}{\longrightarrow} \bigoplus_{E \in \mathcal{E}_{2}^{\prime \prime}} \operatorname{Hom}_{\mathrm{Aut}_{G}(E)}\left(\operatorname{St}_{E}, F\left(C_{G}(E) / E\right)\right) \\
\longrightarrow \bar{\delta}^{1} \longrightarrow \bigoplus_{E \in \mathcal{E}_{3}^{\prime \prime}} \operatorname{Hom}_{\mathrm{Aut}_{G}(E)}\left(\operatorname{St}_{E}, F\left(C_{G}(E) / E\right)\right) \longrightarrow \cdots
\end{aligned}
$$


by removing the terms appearing in the short exact sequences of the form given in Case 1 considered in the proof of Theorem 6.8. Note that if a short exact sequence of modules appears as a subcomplex of a chain complex, removing the modules of this short exact sequence from the chain complex does not change the homology group of the chain complex. Assume also that the short exact sequences of the form given in (6) earlier are removed for every $E \in \mathcal{E}_{2}^{\prime}$ and $E \in \mathcal{E}_{3}^{\prime}$ satisfying $E \leq H=C_{G}(K)$.

Finally assume that maps of the form

$$
F\left(C_{G}(C) / C\right) \rightarrow F\left(C_{G}(C) / C Z\right)
$$

are also removed for all $C$ such that $C Z$ is a maximal rank 2 elementary abelian subgroup such that $C Z \notin \mathcal{B}(G)$. Note that these maps are surjective maps with kernel giving the isomorphism $\widetilde{H}^{0}\left(\mathcal{D}_{G}^{*} ; F\right) \cong \widetilde{H}^{0}\left(\mathcal{A}_{\geq 2}(G) ; A\right)$. So removing these does not change the cohomology groups of the chain complex at dimension 1 .

What is left in $\mathcal{E}_{1}^{\prime \prime}$ are the $G$-conjugacy classes of subgroups $C$ such that $E=C Z$ belongs to the big component $\mathcal{B}(G)$, but $C \not \leq H$. These are the subgroups considered in Case 2 in the proof of Theorem 6.8. Note that the only subgroups left in $\mathcal{E}_{2}^{\prime \prime}$ are the ones that belong to the big component $\mathcal{B}(G)$ and satisfy $E \not \& H$. Recall that for each $C \in \mathcal{E}_{1}^{\prime \prime}$ there is a chain complex of the form

$$
\begin{aligned}
0 \longrightarrow F\left(\bar{C}_{G}(C)\right) \longrightarrow \bigoplus_{\bar{D} \in \mathcal{T}_{1}} F\left(C_{\bar{C}_{G}(C)}(\bar{D}) / \bar{D}\right) \\
\longrightarrow \bigoplus_{\bar{E} \in \mathcal{T}_{2}} \operatorname{Hom}_{\operatorname{Aut}_{\bar{C}_{G}(C)}(\bar{E})}\left(\operatorname{St}_{\bar{E}}, F\left(C_{\bar{C}_{G}(C)}(\bar{E}) / \bar{E}\right)\right) \longrightarrow \cdots
\end{aligned}
$$

which is the chain complex $C_{\mathrm{St}}^{*}(F)$ for the group $\bar{C}_{G}(C)=C_{G}(C) / C$. By taking the direct sum of these chain complexes over all $C \in \mathcal{E}_{2}^{\prime}$, we obtain the chain complex given above in low dimensions with the same maps as $\bar{\delta}^{0}$ and $\bar{\delta}^{1}$. From this we can conclude that

$$
H^{1}\left(\mathcal{D}_{G}^{*} ; F\right) \cong \bigoplus_{C \in \mathcal{E}_{1}^{\prime \prime}} \widetilde{H}^{0}\left(\mathcal{D}_{\bar{C}_{G}(C)}^{*} ; F\right) \cong \bigoplus_{C \in \mathcal{E}_{1}^{\prime \prime}} \widetilde{H}^{0}\left(\mathcal{A}_{\geq 2}\left(\bar{C}_{G}(C)\right) / \bar{C}_{G}(C) ; A\right)
$$

Note that the group $\bar{C}_{G}(C)$ has $\bar{Z}=C Z / C$ as a central subgroup, and the poset $\mathcal{A}_{\geq 2}\left(\bar{C}_{G}(C)\right)$ is equivariantly homotopy equivalent to the poset of elementary abelian subgroups $\bar{E}$ of $\bar{C}_{G}(C)$ such that $\bar{Z}<\bar{E}$. Let $X_{C}$ denote the poset of elementary abelian subgroups $E \in \mathcal{A}_{\geq 2}(G)$ such that $E>C Z$. We have

$$
\widetilde{H}^{0}\left(\mathcal{A}_{\geq 2}\left(\bar{C}_{G}(C)\right) / \bar{C}_{G}(C) ; A\right) \cong \widetilde{H}^{0}\left(X_{C} / C_{G}(C) ; A\right) .
$$

Also note that there is a 1-1 correspondence between $G$-conjugacy classes of $C \not \leq H$ and $G$-conjugacy classes of subgroups $E=C Z$ with $E \not \leq H$. This is because if $C, C^{\prime} \leq C Z$ 
then $C$ and $C^{\prime}$ are $G$-conjugate (note that if $K=\langle a, z\rangle$, then $[c, a]=c a c^{-1} a^{-1}=z$, so $\left.a c a^{-1}=z^{-1} c\right)$. We conclude that

$$
H^{1}\left(\mathcal{D}_{G}^{*} ; F\right) \cong \bigoplus_{E \in \mathcal{E}_{m}} \widetilde{H}^{0}\left(X_{C} / C_{G}(C) ; A\right)
$$

where $\mathcal{E}_{m}$ is the set of $G$-conjugacy class representatives of elementary abelian subgroups of the form $C Z$ such that $C \not \leq H$ and $C Z$ belongs to the big component of $\mathcal{A}_{\geq 2}(G)$. In fact, if it does not belong to the big component, then $C Z$ is maximal and hence we would have $X_{C}=\emptyset$, so we can actually drop this assumption for the above sum and take $\mathcal{E}_{m}$ as the set of $G$-conjugacy class representatives of rank 2 elementary abelian subgroups $E$ such that $Z \leq E$ and $E \not \leq H$.

Let $X=\mathcal{A}_{\geq 2}(G)$ and $Y \subseteq X$ denote the subposet consisting of all $E \in X$ such that either $E \leq H$ or $\operatorname{rk}(E Z) \geq 3$. It is easy to see that $X-Y$ is a discrete set of rank 2 elementary abelian subgroups $E$ such that $Z \leq E$ and $E \not \leq H$. Note that the poset $Y$ is equivariantly contractible to the point $\{K\}$, so we can now apply an equivariant version of [5, Lemma 6.2] to obtain

$$
H^{1}(X / G ; A) \cong \bigoplus_{E \in \mathcal{E}_{m}} \widetilde{H}^{0}\left(X_{C} / C_{G}(C) ; A\right)
$$

Together with the earlier isomorphism, we conclude that $H^{1}\left(\mathcal{D}_{G} ; F\right)$ is isomorphic to $H^{1}(X / G ; A)$.

Now we are ready to prove Theorem 1.5 stated in the introduction.

Theorem 7.9. Let $G$ be a p-group with $p$ odd. Let $D$ denote the p-biset functor of Dade group. Then there is an exact sequence of abelian groups

$$
0 \rightarrow \operatorname{Obs}(D(G)) \rightarrow H^{1}\left(\mathcal{A}_{\geq 2}(G) / G ; \mathbb{Z}\right) \rightarrow H^{1}\left(\mathcal{A}_{\geq 2}(G) / G ; \mathbb{Z} / 2\right)
$$

where the second map is induced by the $\bmod 2$ reduction $\operatorname{map} \mathbb{Z} \rightarrow \mathbb{Z} / 2$.

Proof. By Proposition 7.6, $\operatorname{Obs}(D(G)) \cong H^{1}\left(\mathcal{D}_{G}^{*} ; C_{b}\right)$. Consider the long exact cohomology sequence associated to the short exact sequence $0 \rightarrow C_{b} \rightarrow R_{\mathbb{Q}}^{*} \rightarrow D_{t}^{\Omega} \rightarrow 0$. Note that since $p$ is odd we have $D_{t}^{\Omega}=D_{t}$. This gives an exact sequence of the form

$$
\cdots \rightarrow \operatorname{Obs}\left(R_{\mathbb{Q}}^{*}(G)\right) \stackrel{\pi^{*}}{\longrightarrow} \operatorname{Obs}\left(D_{t}(G)\right) \rightarrow H^{1}\left(\mathcal{D}_{G}^{*} ; C_{b}\right) \rightarrow H^{1}\left(\mathcal{D}_{G}^{*} ; R_{\mathbb{Q}}^{*}\right) \rightarrow H^{1}\left(\mathcal{D}_{G}^{*} ; D_{t}\right) \rightarrow \cdots
$$

We have shown earlier that the map $\pi^{*}$ is surjective (see Remark 7.4). Hence we obtain a short exact sequence of abelian groups of the form

$$
0 \rightarrow \operatorname{Obs}(D(G)) \rightarrow H^{1}\left(\mathcal{D}_{G}^{*} ; R_{\mathbb{Q}}^{*}\right) \rightarrow H^{1}\left(\mathcal{D}_{G}^{*} ; D_{t}\right)
$$


Note that $\partial R_{\mathbb{Q}}^{*}(H) \cong \mathbb{Z}$ and $\partial D_{t}(H) \cong \mathbb{Z} / 2$ for every $H$ that is cyclic or isomorphic to a generalized quaternion group. Hence the proof follows from Proposition 7.8.

We conclude with the following.

Proposition 7.10. Let $G$ be a p-group with $p$ odd. If $D_{f}$ denotes the quotient functor $D / D_{t}$, then

$$
\operatorname{Obs}\left(D_{f}(G)\right) \cong H^{1}\left(\mathcal{A}_{\geq 2}(G) / G ; \mathbb{Z}\right)
$$

Proof. The exact sequence $0 \rightarrow R_{\mathbb{Q}}^{*} \rightarrow B^{*} \rightarrow D / D_{t} \rightarrow 0$ gives a long exact sequence

$$
\cdots \rightarrow \operatorname{Obs}\left(B^{*}(G)\right) \rightarrow \operatorname{Obs}\left(D_{f}(G)\right) \stackrel{\delta}{\longrightarrow} H^{1}\left(\mathcal{D}_{G}^{*} ; R_{\mathbb{Q}}^{*}\right) \rightarrow H^{1}\left(\mathcal{D}_{G}^{*} ; B^{*}\right) \rightarrow \cdots
$$

By [8, Theorem 3.1], we have $\operatorname{Obs}\left(B^{*}(G)\right)=0$, and by Lemma 4.1 and 7.5, we have

$$
H^{1}\left(\mathcal{D}_{G}^{*} ; B^{*}\right) \cong \operatorname{Ext}_{R \mathcal{D}_{G}}^{1}\left(J, B^{*}\right)=0
$$

hence we obtain $\operatorname{Obs}\left(D_{f}(G)\right) \cong H^{1}\left(\mathcal{D}_{G}^{*} ; R_{\mathbb{Q}}^{*}\right)$. Now the result follows from Proposition 7.8 and from the fact that $\partial R_{\mathbb{Q}}^{*}(H) \cong \mathbb{Z}$ for every $H$ that is cyclic or isomorphic to a generalized quaternion group.

\section{References}

[1] L. Barker, Rhetorical biset functors, rational $p$-biset functors and their semisimplicity in characteristic zero, J. Algebra 319 (2008) 3810-3853.

[2] S. Bouc, Gluing endo-permutation modules, J. Group Theory 12 (2009) 651-678.

[3] S. Bouc, Biset Functors for Finite Groups, Lecture Notes in Mathematics, vol. 1990, Springer-Verlag Berlin Heidelberg, 2010.

[4] S. Bouc, Rational p-biset functors, J. Algebra 319 (2008) 1776-1800.

[5] S. Bouc, N. Mazza, The Dade group of (almost) extraspecial p-groups, J. Pure Appl. Algebra 192 (2004) 21-51.

[6] S. Bouc, J. Thévenaz, Gluing torsion endo-permutation modules, J. Lond. Math. Soc. 78 (2008) $477-501$.

[7] S. Bouc, E. Yalçın, Borel-Smith functions and the Dade group, J. Algebra 311 (2007) 821-839.

[8] O. Coşkun, Gluing Borel-Smith functions and the group of endo-trivial modules, Bull. Lond. Math. Soc. 43 (2011) 912-926.

[9] E.C. Dade, Endo-permutation modules over p-groups, I, II, Ann. of Math. 107 (1978) 459-494 108 (1978) 317-346.

[10] D. Gorenstein, R. Lyons, R. Solomon, The Classification of Finite Simple Groups, Number 2, Mathematical Surveys and Monographs, vol. 40, American Mathematical Society, 1996.

[11] J. Grodal, Higher limits via subgroup complexes, Ann. of Math. 155 (2002) 405-457.

[12] I. Hambleton, L. Taylor, B. Williams, Detection theorems for K-theory and L-theory, J. Pure Appl. Algebra 63 (1990) 247-299.

[13] S. Jackowski, J. Slominska, G-functors, G-posets and homotopy decompositions of $G$-spaces, Fund. Math. 169 (2001) 249-287.

[14] B. Mitchell, Rings with several objects, Adv. Math. 8 (1972) 1-161.

[15] R. Oliver, Higher limits via Steinberg representations, Comm. Algebra 22 (4) (1994) 1381-1393. 
[16] D. Quillen, Homotopy properties of the poset of nontrivial $p$-subgroups of a group, Adv. Math. 28 (1978) 101-128.

[17] P. Webb, Stratifications and Mackey functors II: globally defined Mackey functors, J. K-Theory 6 (2010) 99-170.

[18] C.A. Weibel, An Introduction to Homological Algebra, Cambridge Studies in Advanced Mathematics, vol. 38, Cambridge University Press, 1994. 